\title{
DIE KUNST DER VERFASSUNGGEBUNG
}

\author{
Von Herbert KrüGER
}

Es gibt heute kaum noch eine schöpferische Leistung im Bereich der Gesellschaft, die "naiv" hervorgebracht werden könnte -, die also ihre Entstehung einem „Originalgenie“ ausschließlich und allein verdankte. Überall ist vielmehr eine solche Hervorbringung mehr oder weniger „rationalisiert", indem der Schöpfer hierfür gebildet wird, indem ihm Helfer zuarbeiten usw. usw. - was alles sich nicht zuletzt in Kollektivierung äußert: Nicht mehr einer Person, sondern einer Kommission, einer Versammlung und dergl. hat man infolgedessen die Schaffung einer Verfassung oder von Gesetzen zuzuschreiben.

Mit einem Wort: „Natur" wird auch an dieser Stelle immer mehr durch „Kunst“ verdrängt. Damit ergibt sich die Notwendigkeit von „Kunstlehren“ auch im Bereich von Staat und Verfassung. Etwas Derartiges ist nicht so neu wie es auf den ersten Blick wirken mag. Die Fürsten der Neuzeit z. B. wurden nicht nur darüber unterrichtet, was einen guten Fürsten ausmache und wie er sich zu verhalten habe -, daß es ihm etwa gut zu Gesichte stehe, wenn er Lesen und Schreiben beherrsche und sich allzu vielen Alkohols enthalte - , sie konnten sich auch aus einer unübersehbaren „Staats-Räson“ Literatur unterrichten, wie man erfolgreich regieren könne ${ }^{1}$ : Es mag sich um eine Literaturgattung handeln, an die die heutige „Regierungslehre“ anknüpft.

Unter solchen Umständen dürfte es an einer Kunstlehre für den Verfassungọgeber (oder unpersönlich: Die Verfassunggebende Gewalt) an sich nicht fehlen. Zwar gibt es seit langem Untersuchungen, die der „Kunst der Gesetzgebung“ gewidmet sind $^{2}$-, aber es scheint als ob über die Kunst der Verfassunggebung allenfalls beiläufig etwas gesagt worden wäre. Es mag dies daran liegen, daß Verfassungen durchweg in Eile, unter Druck oder ähnlichen Zwängen hervorgebracht werden müssen, die für umständliche theoretische, insbesondere Verfahrungsüberlegungen keine Zeit lassen. Um so weniger sollte sich daher gerade auch die Verfassungsvergleichung, wie sie diese Zeitschrift pflegt, die Möglichkeit entgehen lassen, ihre Aufmerksamkeit der Schöpfung einer neuen Gesamtverfassung zu widmen, wie sie jetzt in der Schweiz mit bewunderungswürdiger Umsicht und vorbildlicher Sorgfalt betrieben wird. Zur Zeit ist man dort so weit gediehen, daß die Arbeitsgruppe für die Vorbereitung einer Totalrevision der Bundesverfassung, die unter dem Vorsitz von Alt Bundesrat Prof. Dr. F. T. Wahlen gewirkt hat, einen „Schlußbericht" vorgelegt hat, den sog. Wahlen-Bericht ${ }^{3}$. Die Fortsetzung der Arbeiten ist nunmehr einer „Expertenkommission für die Totalrevision des Bundesverfassung " aufgetragen worden: Sie besteht aus 46 Mitgliedern, steht unter dem Vorsitz von Bundesrat Kurt Furgler und hat am 9. Mai 1974 ihre Tätigkeit aufgenommen4. Sie will bis Mitte August einen ersten formulierten Rohentwurf vorlegen ${ }^{5}$. Der Widerhall, den die Leistung des Wahlen-Ausschusses in der Schweiz und im Aus-

$1 \mathrm{Als}$ Beispiel sei genannt die Principis Christiani Institutio Per Aphorismos digesta Auctore Desiderio Erasmo Roderodamo. Lugduni Batavorum (= Leyden) 1641.

2 Vgl. zuletzt etwa Uwe Krüger, Der Adressat des Rechtsgesetzes - ein Beitrag zur Gesetzgebungslehre (Berlin 1969) vor allem S. $82 \mathrm{ff}$

3780 Seiten (Bern 1973).

4 Vgl. Neue Zürcher Zeitung vom 17. März und 11. Mai 1974.

5 NZZ vom 13. Mai 1974. 
land gefunden hat ${ }^{6}$, scheint mir ihrer Bedeutung nicht voll gerecht $\mathrm{zu}$ werden: Es handelt sich in meinen Augen um ein Werk, das geradezu als eine sachnahe, kundige, das Pro und Kontra aller Probleme erschöpfende Verfassungslehre angesehen werden darf. Vor allem aber hat sich der Ausschuß eine Kunstlehre der Verfassunggebung zwar nicht expressis verbis erarbeitet, man darf aber gewiß dasjenige seiner Darlegungen als eine solche ansehen, was er als „Erster Teil: Voraussetzungen einer Totalrevision der Bundesverfassung" seinem Schlußbericht vorausschickt. Schon angesichts der Seltenheit einer solchen Leistung sollte man sie festhalten und auswerten.

Der Versuch, die Umrisse einer Kunstlehre der Verfassunggebung zu zeichnen, müßte sich in erster Linie mit dem Verfahren beschäftigen, das man dieserhalb einzuschlagen hat. Hierzu gehört vor allem wieder die Frage, welcher Art von Personen man dieses Werk anvertrauen soll: „Staatsmännern“ oder „Experten ". Wenn nämlich eine Verfassung nicht nur einen richtigen Inhalt aufweisen, sondern auch das Volk ansprechen, ja begeistern soll, dann mag ein Überwiegen des Expertentums zwar größere inhaltliche Richtigkeit in Aussicht stellen (obwohl dies keineswegs sicher ist), aber diese Richtigkeit mag erkauft sein durch eine Blässe des Gedankens und eine Fachlichkeit der Sprache, die Integration zur Einheit gewiß nicht fördert, weil sich nur Gelehrte oder Fachleute von einer solchen Verfassung angesprochen fühlen können. Gerade auch die Verfassunggebung innerhalb einer Demokratie wird sich solcher nicht-juristischer Überlegungen nicht entschlagen können: Hier wird die Verfassunggebung vom Volk ausgehen (etwa: Wahl einer Verfassunggebenden Versammlung) wie wegen der entscheidenden $\mathrm{Zu}$ stimmung an dasselbe zurückgelangen müssen (Unterbreitung des Textes unter Volksabstimmung).

Dennoch sieht sich der Verfasser außerstande, auch und vor allem das Verfahren der Verfassunggebung in diesem Versuch einzubeziehen. Er sieht sich zu dieser Unterlassung gezwungen durch den Umstand, daß es hierzu eines unendlichen Tatsachenmaterials bedürfte: Bevor man sich darüber ausläßt, wie man es machen sollte, muß man hier wissen, wie man es in einer Unzahl von Fällen gemacht hat. Dazu fehlt es jedoch an ausreichender Vorarbeit: Spezifische und eingehende Darstellungen konkreter Verfassunggebungen sind jedoch relativ selten ${ }^{7}$, und von Vergleichung solcher Prozesse kann noch weniger die Rede sein. Verhältnismäßig am stärksten hat in diesem Rahmen die Aufmerksamkeit auf sich gezogen die Frage der Aktivlegitimation zu Verfassunggebung - die Frage nach dem „Pouvoir Constituant ${ }^{8 “}$.

Nur berührt werden sollen alle Probleme, die mit der rechten Gestalt einer Verfassung zusammenhängen. Hier scheint man sich immerhin über zweierlei einig. Was den Umfang angeht, so sollte eine Verfassung kurz sein ${ }^{9}$, damit sie nicht

6 Vgl. NZZ vom 25. November 1973: „Der am 6. September veröffentlichte Schlußbericht . . . hat bisher nicht jenen Widerhall gefunden, den man dem Gewicht dieser Sache gemäß eigentlich hätte erwarten dürfen."

7 Als wirklich gelungen sind mir nur zwei Darstellungen bekannt, nämlich Heinrich Triepel, Zur Vorgeschichte der Norddeutschen Bundesverfassung, in: Festschrift für Otto Gierke (Weimar 1911); Granville Austin, The Indian Constitution - Cornerstone of a Nation - (Bombay Oxford UP 1972). Beachtenswert scheinen mir u. a. ferner: Bernard Gilson, La Découverte du Régime Présidential (Paris 1968) (Für die Vereinigten Staaten); G. W. Choudhury, Constitutional Development in Pakistan 2. Ed. (London Longman 1969); A. Jeyratnam Wilson, Politics in Sri Lanka, 1947-1973 (London 1974 Macmillan), S. 234 ff.; J. Nescher, Uruguay - Besonderheiten eines Verfassungssystems (Berlin 1972) S. $91 \mathrm{ff}$.; J. A. La Nanza, The Making of the Australian Constitution (Melbourne UP 1972).

8 Hierzu zuletzt etwa Udo Steiner, Verfassung und Verfassunggebende Gewalt des Volkes (Berlin 1966) und G. Burdeau, Traité de Science Politique IV, 2. Aufl. (Paris 1969), S. $181 \mathrm{ff}$.

9 Vgl. hierzu etwa Th. Oppermann, Kulturverwaltungsrecht (1972), S. 170, C. Tomuschat, Verfassungsgewohnheitsrecht? (Heidelberg 1972), S. 50. Vor allem aber Wahlen-Bericht (vgl. oben Anm. 3), S. 34: "Die Dauerhaftigkeit weist aut Knappheit und Kargheit in Festlegung und Wortlaut." 
schon deswegen von Volk zurückgewiesen wird. Ihre Sätze sollten ferner, und zwar selbst zu Lasten der juristischen Präzision, für jedermann verständlich und gewinnend $\operatorname{sein}^{10}$. Die Sätze einer Verfassung sollen ferner elastisch sein ${ }^{11}$, ihre Begriffe daher sich mit der Zeit wandeln können und dürfen ${ }^{12}$, und eine Verfassungsänderung sollte nicht allzusehr erschwert werden ${ }^{\mathbf{1 3}}$.

Die nachfolgenden Bemühungen konzentrierten sich hiernach auf alles, was mit dem Inhalt einer Verfassung zusammenhängt, und versuchen zu ergründen, was die beste Richtigkeit eines solchen Inhaltes ausmacht. Hier wird vor allem, wie jetzt schon geságt sei, herauszuarbeiten sein, daß eine Verfassung nicht, ja nicht einmal vorwiegend eine Verwirklichung von Idealen sein kann -, daß sie zudem und gerade in einem solchen Falle darauf bedacht sein muß, „daß gerade die Leistungs- und Funktionsfähigkeit einer Verfassung zu ihren wichtigsten Legitimi-

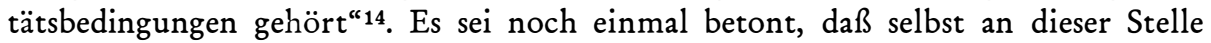
nicht mehr erwartet werden kann als ein Versuch, dessen Ergebnisse noch vorläufiger und anfechtbarer sind als diejenigen wissenschaftlicher Bemühungen, die auf bereits beacktertem Boden zu ernten suchen.

\section{Die Situation}

Man kann nicht ganz sicher sein, daß nicht der alte Traum von der absolut besten Verfassung ebenso wie die Idee vom vollkommenen Staat in neuer Gestalt wiederaufgelebt sind: Es ist die Herrschaft von Ideologien in Ost und West, die eine solche Befürchtung begründet. Diese Beobachtung ist gerade für die neuen Staaten in Úbersee wichtig. Liest man z. B. ${ }^{15}$, daß der für sie einzig richtige Weg zur Modernisierung der sozialistisch-kommunistische sei, dann werden sie hiermit zu einer Politik gedrängt, die die ein für allemal und überall, also einzig richtige sein soll - nicht anders als es eine Lehre von der besten Verfassung und vom vollkommenen Staat tun würden. Da eine solche absolute Richtigkeit natürlich durch nichts bewiesen und vor allem nicht beweisbar ist, handelt es sich darum, einer allenfalls relativen Richtigkeit Ausdehnung über ihren eigentümlichen Bereich hinaus zu verschaffen -, also um ideologischen Imperialismus.

$10 \mathrm{Vgl}$. hierzu Y. P. Ghai \& J. P. W. B. McAuslan, Public Law and Political Change in Kenya (Nairobi Oxford UP 1970), S. 210.

11 Vgl. hierzu A. de Tocqueville, Souvenirs (= Tome XII der Oeuvres Complètes Paris 1964) S. 191: "Je pensais, depuis longtemps, qu'au lieu de viser à rendre nos gouvernements éternels, il fallait tendre à ce qu'on pût les changer d'une manière facile et regulière."

12 Diese Möglichkeit verschließt einer Verfassung die sog. "Versteinerungstheorie ${ }^{*}$ des Osterreichischen Verfassungsgerichtshofes: Ihr zufolge behalten die, in den Verfassungssätzen enthaltenen Begriffe auf alle Ewigkeit jene Bedeutung, die ihnen im Augenblick der Verfassunggebung innewohnte; vgl. hierzu Norbert Wimmer, Materiales Verfassungsverständnis (= Forschungen aus Staat und Recht 15), Wien und New York (1971), S. $32 \mathrm{ff}$.

13 Zum Thema Verfassungsrevision sei folgende Literatur genannt: W. Strauß, U. Scheuner, A. Hollerbach, Totalrevision des Grundgesetzes? = Nr. 24 der. Veröffentlichungen der Katholischen Akademie der Erzdiözese Freiburg i. Br. (1971). Hans Huber, Die Gesamtänderung der Verfassung. Ansätze für einen Vergleich zwischen Osterreich, der Schweiz und der Bundesrepublik Deutschland, in: Festschrift für Ulrich Scheuner (Berlin 1973), S. $183 \mathrm{ff}$. P. Saladin, Die Kunst der Verfassungserneuerung, in: Gedenkschrift für M. Imboden (1972), S. 269 ff. K. Stern, Totalrevision des Grundgesetzes?, in: Festgabe für Theodor Maunz (München 1971), S. $391 \mathrm{ff}$. Werner Weber, Das Problem einer Revision und gabe für Theodor Maunz (München 1971), S. $391 \mathrm{ff}$. Werner Weber, Das Problem einer Revision und
einer Totalrevision des Grundgesetzes, in: Festgabe für Theodor Maunz (München 1971), S. 450 ff. einer Totalrevision des Grundgesetzes, in: Festgabe für Theodor Maunz (München 1971), S. 450 ff.
Aus der Praxis vgl. "Zwischenbericht der Enquête-Kommission zu Fragen der Verfassungsreform gemäß Beschluß des Deutschen Bundestages Drucksache VI/1211, in: Deutscher Bundestag 6. Wahlperiode Drucksache VI/3829 (auch in: Zur Sache 1/73 Themen Parlamentarischer Beratung, herausgegeben vom Presse- und Informationszentrum des Deutschen Bundestages).

14 So Hans-Peter Schneider, Die Parlamentarische Opposition im Verfassungsrecht der Bundesrepublik Deutschland (Frankfurt am Main 1974) I, S. 163.

$15 \mathrm{Vgl}$. Günter Bartel, Industrialization in the Arab Countries of the Middle East (Berlin 1972) und G. Brehme u. a. (Redaktionskollegium), Partei und Staat in den Ländern mit sozialistischer Orientierung (Berlin 1974). 
$\mathrm{Da}$ die neuen Völker in Übersee den kolonialen Imperialismus gewiß nicht abgeschüttelt haben, um sich einem ideologischen Imperialismus zu unterwerfen, gilt es schon an dieser Stelle entschieden hervorzuheben, daß Verfassungen nicht aus Ideologien, Prinzipien, Begriffen und ähnlich abstrakten Quellen gegeben werden dürfen - daß vielmehr Verfassunggebung sich in Zeit und Raum vollzieht und vollziehen muß, wenn eine Verfassung hic et nunc das Richtige treffen und das von ihr erwartete leisten soll. Nicht zuletzt gilt dies für die Frage, was ein legitimer Anstoß zu Verfassunggebung sein $\mathrm{kann}^{16}$. Illegitim sind in dieser Hinsicht hiernach und zwar insbesondere dann, wenn man dem „Prinzip der Nicht-Identifikation " folgt ${ }^{17}$ der Wille, einer Ideologie und dergl., zum Durchbruch zu verhelfen, eine bestehende Verfassung im Sinne einer Ideologie zu „verbessern“ oder, von einer Verfasng proklamierte Grundsätze besser zu verwirklichen oder gar zu ausschließlicher Herrschaft zu erheben. Überall ist somit der „Raserei der Vernunft" (G. F. W. Hegel) zu wehren sowie an "Situation“ und „Lagen“ anzuknüpfen.

\section{Die Situation als „rechte Zeit“" der Verfassunggebung18}

Verfassunggebung kann offensichtlich nicht die Sache aller Tage, des Alltags sein. F. C. von Savigny ${ }^{19}$ hat Gesetzgebung nur einer Zeit zugestanden, die einen "Beruf" für ein solches Werk habe, und bekanntlich seiner Zeit eine solche Berufenheit abgesprochen. Ist etwas Derartiges im Grunde geschichtsphilosophisch gemeint, so kann man eine solche These auch rationalisieren: So hat z. B. Nehru gemeint, es sei nicht die rechte Zeit für eine Verfassunggebung, wenn die Leidenschaften des Volkes aufgewühlt seien ${ }^{20}$. In diesem Zusammenhang wird in der Schweiz vielfach die Auffassung vertreten, daß Zeiten „eines unerhört breiten, tiefgreifenden und raschen Wandels" nicht geeignet seien, eine gute Verfassung hervorzubringen ${ }^{21}$. K. Stern ${ }^{22}$ wiederum meint: „Der ,Beruf' sowie der kühle Verstand und der lange Atem unserer Zeit für eine Verfassungsreform sind angesichts der starken innen- und außenpolitischen Konfrontation nicht nur der staatlichen Organe derzeit zumindest zweifelhaft.“

Wenn eine Verfassung vor allem auch die Veranstaltung eines Volkes gegen sich selbst ist -, wenn es also Höhepunkte seiner Geschichte mittels Verfassunggebung auf Tiefpunkte erstrecken und diese dadurch überwinden oder wenigstens abschwächen will23, dann ergibt sich aus solchem Sinn einer Verfassung, daß sie in der Regel nicht an einem Tiefpunkt, sondern an einem Höhepunkt der nationalen

16 Vgl. hierzu K. von Beyme, Die verfassunggebende Gewalt des Volkes (Tübingen 1968), S. 54.

17 Vgl. Herbert Krüger, Allgemeine Staatslehre, 2. Aufl. (Stuttgart 1966), S. $178 \mathrm{ff}$.

18 Hierzu Friedrich Schiller: „Es gibt im Staatenleben Augenblicke, wo er dem Weltgeist näher ist als sonst, und eine Frage frei hat an das Schicksal." In diesem Sinne muß auch ein Verfassunggeber

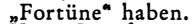

19 Vom Beruf unjerer Zeit für Gesetzgebung und Recht, zuerst 1814; vgl. heute etwa W. Kewenig, Verfassungsreform - ein Beruf unserer Zeit? - in: Die Offentliche Verwaltung 1971, S. $524 \mathrm{ff}$.

20 Nach Austin a. a. O. (oben Anm. 7) S. 243.

21. Wahlen-Bericht (oben Anm. 3) S. 47.

22 a. a. O. (oben Anm. 13), S. 398; vgl. hierzu auch Werner Weber, Das Problem der Revision des Grundgesetzes, in: Festgabe für Theodor Maunz (München 1971), S. 451 ff. S. 461, der in einer Sammelveröffentlichung einer größeren Zahl namhafter Schweizer Rechtsgelehrter erhebliche Zweifel hinsichtlich der Opportunität und der zeitlichen Reife der beabsichtigten Totalrevision gefunden haben will.

$23 \mathrm{Vgl}$. hierzu J. Bryce (nach Stern a. a. O. S. 404): „Eine Konstitution verkörpert aber gleichzeitig mit dem Grundsatz der Freiheit auch den Grundsatz der Selbstbeherrschung. Das Volk hat beschlossen, bestimmte Normen außerhalb der Reichweite nur temporärer Impulse, die aus der Leidenschaft oder der Laune entspringen, zu setzen und diese Normen zum dauernden Ausdruck seines ruhigen Nachdenkens und seiner wohlüberlegten $Z$ weckeinstellung $\mathrm{zu}$ machen .. “. Im gleichen Sinne Herbert Krüger, Artikel „Verfassung ${ }^{\alpha}$, in: Handwörterbuch der Sozialwissenschaften Band 11 (1961), S. 72 ff.; jetzt in: Staat - Wirtschaft - Völkergemeinschaft (Frankfurt am Main 1970), S. 160 ff. 
Geschichte gegeben werden sollte. So richtig dies alles ist, so liegt die eigentliche Schwierigkeit in der Unmöglichkeit, festzustellen, ob man sich auf einem solchen Höhepunkt befindet, insbesondere ob es sich um eine Zeit handelt, die die Vorsehung mit einem Beruf für Verfassung- und Gesetzgebung begnadet hat. Im Grunde läßt sich etwas Derartiges nicht im voraus, sondern erst aus dem Ergebnis feststellen: Das Warten auf einen fruchtbaren Höhepunkt des nationalen Genies mag bewirken, daß niemals eine Verfassung zustande kommt oder daß gerade der falsche Zeitpunkt gewählt wird ${ }^{24}$.

\section{Die „Situation“ als die Gesamtheit der Umstände, unter denen eine Verfassung gegeben wird}

Mit diesem Thema ist danach gefragt, wie der Verfassunggeber sich angesichts der Umstände, in denen er sich vorfindet, verhalten sollte - insbesondere aber welche Umstände die Schöpfung einer guten Verfassung befördern und welche das Gegenteil in Aussicht stellen. Eine Kunstlehre der Verfassunggebung wird versuchen müsse, solche Konstellationen zu typisieren. Der Wahlen-Bericht ${ }^{25}$ unterscheidet unter diesem Blickwinkel: „Der ganze zweite Teil dieses Berichts ist beherrscht von dem Eindruck, daß es einen wesentlichen, wiewohl begrifflich nicht scharf abgrenzbaren Unterschied ausmacht, ob durch die Verfassunggebung auf der einen Seite eine tiefschürfende politische Krise (z. B. Revolution) beendet und eine neue staatliche Gemeinschaft erst aufgebaut werden soll oder ob auf der anderen Seite eine existenz- und funktionsfähige Gemeinschaft sich eine bessere Grundordnung zu geben trachtet."

a) Der erste Typ, den „räumliche“ Situationen aufweisen mögen, unter denen eine Verfassung gegeben wird, mag dadurch gekennzeichnet werden, daß der Verfassunggeber nichts vorfindet, weil entweder von vornherein nichts vorhanden war oder eine Revolution „Tabula rasa" geschaffen hat. Hier hat eine Verfassung offensichtlich nicht nur zu verfassen, sondern zu gründen, und zwar einen neuen Staat zu gründen ${ }^{26}$. In solchen Fällen kommt es gar nicht erst $\mathrm{zu}$ der vielfach erörterten Frage, ob und inwieweit Kontinuität ein Gebot für die Verfassunggebende Gewalt darstellt. Mit alledem ist jedoch nicht ausgemacht, daß hier Zeit und Raum ipso facto unbeachtlich zu sein hätten. Man denke etwa an eine nationale Katastrophe, insbesondere eine totale Niederlage, der zudem eine langjährige Herrschaft der Sieger folgt. Hier muß zweifellos erst einmal ein Mindestmaß von politischer Ordnung geschaffen werden. Auf der anderen Seite ist dies gewiß nicht die Situation, die die Schaffung von vollständigem, vollkommenem und endgültigem begünstigt. Die Lösung, die sich in einer solchen Antinomie vorzeichnet, ist das Provisorium, das inhaltlich gesehen vor allem die Nationale Erneuerung einzuleiten hat. Wie die Praxis in einem solchen Falle aussehen mag, zeigt das Beispiel der Bundesrepublik Deutschland: Ihr "Grundgesetz" von 1949, das als Provisorium konzipiert war,

24 Vgl. hierzu R. von Mohl, Constitutionelle Erfahrungen - Ein Beitrag zur Verfassungspolitik $\overline{-}$, in: Staatsrecht, Völkerrecht und Politik I (1860), S. 322 ff., S. 323: Er verteidigt die Naivität von Verfassunggebern, „weil vielleicht mehr Bewußtsein es gar nicht hätte zu der Tat kommen lassen, mit welcher man doch in der Hauptsache zufrieden zu sein Ursache hat".

25 S. 20

26 Womit nicht verneint sein soll, daß auch die Verfassung eines gegebenen Staates stets ein Stück von Staatsgründung enthält, indem sie eine solche programmiert. 
wurde dennoch sogleich als eine vollständige Verfassung gestaltet und hat sich auf die Dauer auch den Charakter der Endgültigkeit erworben ${ }^{27}$. Eine Auseinandersetzung mit der Situation findet sich lediglich in Spuren: Im großen und ganzen handelt es sich um eine Verfassung, wie sie sich jedes andere Volk ohne Rücksicht auf die Situation auch hätte geben können.

Handelt es sich um die gegenteilige Situation, hat also ein Volk gesiegt, und zwar vor allem in einem nationalen Befreiungskampf gesiegt, dann wird eine solche Situation von der Verfassung Vorkehrungen dafür fordern, daß der Sieg insbesondere von dem Besiegten nicht mehr in Frage gestellt werden kann. Einer solchen Reaktion verdankt die Verfassung der USA die starke Stellung des Präsidenten ${ }^{28}$.

$\mathrm{Zu}$ demjenigen Typus von „Befindlichkeit“, der hiermit angesprochen wird, scheinen mir auch die Neuen Nationen in Übersee nach Erlangung der Souveränität zu gehören. Mögen sie im einzelnen auch mehr aus der Kolonialzeit fortgeführt haben als man gemeinhin annimmt - , so mußte doch im entscheidenden neu begonnen werden: Mit der Konstituierung der Nation und ihrer Staatlichkeit. Bekanntlich haben sich hierbei die frankophonen Staaten an der Französischen Verfassung von 1958, die anglophonen am "Westminster-Modell“ orientiert ${ }^{29}$. Man muß jedoch daran zweifeln, ob dies die eigentliche Konstituierung darstellt, ob man sie nicht in anderen Setzungen zu suchen hat, die praeterkonstitutionell, aber anders als die Texte eine Auseinandersetzung mit der vorgefundenen Situation darstellen. Es handelt sich dabei um drei Größen, die die Einheit von Nation und Staat gewissermaßen als Selbstzweck verkörpern: Die eine Partei ${ }^{30}$ und die eine Armee ${ }^{31}$ und der eine Öffentliche Dienst. Es heißt den Zusammenhang von Situation und Verfassunggebung gründlich verkennen, wenn man solche Lösungen als „undemokratisch", „unfreiheitlich" usw. verurteilt: Wenn es sich zunächst und vor allem um Einheit im existentiellsten Sinne des Wortes handelt, kann man nicht pluralistisch beginnen. Die europäische Geschichte ist übrigens in der entsprechenden Situation nicht anders verfahren, wie das Zeitalter des Absolutismus zeigt.

Ein qualifiziertes Beispiel dafür, daß eine Verfassung nach Gewinnung der nationalen Einheit vor allem auf Einigung bedacht ist und daß sie hierfür eine ingeniöse Vorkehrung trifft, bietet die Verfassung des Deutschen Reiches von 1871: Hier wird die föderalistische Institution des Gesamtstaates, der Bundesrat, in einem solchen Maße mit Zuständigkeiten und damit mit Verantwortlichkeit für den $\mathrm{Ge}-$ samtstaat ausgestattet, daß der Föderalismus zum Vehikel des Unitarismus wird ${ }^{32}$.

b) die zweite Alternative, die in diesem Zusammenhang, der Wahlen-Bericht kennt, wird dadurch gekennzeichnet, daß der Verfassungsgeber ein einiges und geordnetes Gemeinwesen vorfindet, und daß es sich somit lediglich um Veränderung,

27 Vgl. hierzu H. P. Ipsen, Ưber das Grundgesetz - nach 25 Jahren -, in: Die Offentliche Verwaltung (1974) S. $289 \mathrm{ff}$.

28 Vgl. Gilson a. a. O. (oben Anm. 7), S. 142, 149.

29 Solche Rezeptionen stehen unter der Hoffnung, sie würden die eigenständige Lösung night überlagern oder gar ausschließen, sondern ihr im Gegenteil zu besserer Gestalt und richtigem Gehalt ju $^{2}$ verhelfen es ließe sich m. a. W. das gute alte und das fremde neue zu traditioneller und moderner Etgenheit verbinden; vgl. hierzu J. R. Levenson, Confucian China and its Modern Fate: A Trilogy (California UP 1968), S. $49 \mathrm{ff}$.

30 Vgl. hierzu u. a. Ahmed Mahiou, L'Avenement du Parti unique en Afrique Noire (Paris 1969), und Hartwig Rogge, Die Verfassung des afrikanischen Einparteienstaates (Hamburg 1974).

$31 \mathrm{Vgl}$. hierzu vor allem im allgemeinen Alfred Stepan, The Military in Politics - Changing Patterns in Brazil - (Princeton UP 1971), im besonderen Robin Luckham, The Nigerian Military - A Sociological Analysis of Authority and Revolt 1960-67 (Cambridge UP 1971).

$32 \mathrm{Vgl}$. hierzu Hermann Rehm, Unitarismus und Föderalismus in der Deutschen Reichsverfassung (Dresden 1898) S. 29: Bismarck „räumte dem Bundesrat den größten rechtlichen Einfluß im Bundesorganismus ein, legte in seine Hand den größten rechtlichen Teil der Bundesgewalt, um so die Garantie zu schaffen, daß die Bundesglieder gern dem Bundesverband angehörten. Nicht das Gefühl nur von Beherrschten, sondern von Mitherrschern sollten die Bundesfürsten haben .... 
nicht um Gründung handelt. In einem solchen Falle stellt sich als erste die Frage, wie weit und wie tief der Verfassungsgeber mit seinen Veränderungen gehen darf, insbesondere, ob eine Totalrevision nur revolutionär oder auch konstitutionell möglich ist. Das zweite wird ausgeschlossen von jener Theorie, die einer jeden Verfassung einen Kern zuschreibt, der von der "Gesamt-Entscheidung (sc des pouvoir constituant) über Art und Form der Politischen Einheit ${ }^{33}$ " gebildet wird. Wenn man sich die Einzelheiten des revolutionären Aktes ausmalt, der eine solche Gesamt-Entscheidung fällt, insbesondere auch die Wahrscheinlichkeit schrecklichen Blutvergießens, dann wird man es vorziehen, heute jener Logik nicht mehr zu folgen ${ }^{33 a}$.

Erweist sich somit auch eine Verfassungsänderung als legal, die in der Sache und nach ihrer Gewichtigkeit auf die Gebung einer grundlegend neuen Verfassung hinausläuft, dann erscheinen zwei Spielarten dieser Möglichkeit als denkbar.

aa) Einmal mag es die Wirklichkeit sein, die nach einer solchen Verfassungsgebung drängt. Wenn z. B. die Futurologie den Eintritt revolutionärer Fakten (z. B. Erschöpfung der Rohstoffvorräte) oder radikal anderer Tendenzen (etwa das Erscheinen einer "postindustriellen Gesellschaft ${ }^{34}$ “ mit Sicherheit voraussagen würde - , und wenn man es nicht darauf ankómmen lassen wollte, daß solche neuen Daten sich als Katastrophe oder als Bürgerkrieg zur Geltung bringen, dann wird der Verfassunggeber nicht umhin können, die Nation auch von Verfassungs wegen auf solche Möglichkeiten vorzubereiten und deren glatte und friedliche Bewältigung möglich $z u$ machen. Im Grunde genommen ist ja der Moderne Staat ohnehin konzipiert und konstruiert als eine Institution, die gerade auch auf das Unerwartete und Unvorhersehbare gewappnet sein sollte. Folgerichtig müßte jede Verfassung per se eine Notstandsverfassung sein. Seltsamerweise ist es jedoch gerade der Verfassungsstaat, der Verfassung und Staat zugunsten von Freiheit und Sicherheit der Bürger im einzelnen entwaffnet hat, so daß das Unerwartete und Unvorhersehbare sich in eine besondere Notstandsverfassung hat zurückziehen müssen, was natürlich nicht ohne Verminderung der Verteidigungs- und Bewältigungskraft abgehen konnte. Letzten Endes hat man hierfür den Optimismus verantwortlich zu machen, dem sich die spätliberale Freiheit verschrieben hat: Angesichts der von diesem Optimismus selbst ausgelösten Entwicklungen insbesondere technischer Art scheint allerdings eine gewisse Ernüchterung geboten, von der sich insbesondere der Verfassunggeber nicht ausschließen sollte ${ }^{35}$.

Man darf nicht von vornherein ausschließen ${ }^{36}$, daß angesichts einer solchen Zukunft der Verfassunggeber ins Auge fassen müßte einen Vorrang der Regierung, die sich nicht allein auf eine Volksvertretung, sondern auch und vor allem auf Experten stützt - wobei man sich wahrscheinlich noch glücklich schätzen könnte, wenn diese Regierung sich nicht als Militärregierung darstellt. Offensichtlich entspricht ein solches Bild nicht dem klassischen Modell des "Verfassungsstaates". Aber offenbar kann man sich den Folgen nicht entziehen,

33 So Carl Schmitt, Verfassungslehre (München und Leipzig 1928), S. 20.

33a Das Grundgesetz der BRD hat es in Art. 79 III dennoch getan.

34 Vgl. hierzu S. P. Huntington, Postindustrial Politics; How Benign Will It Be?, in: Comparative Politics 6 (1973), S. $5 \mathrm{ff}$

35 Vgl. hierzu auch Herbert Krüger, Die Modernität des Modernen Staates, in dieser Zeitschrift 6 VRU (1973), S. 5 f.

36 Vgl. zum Folgenden G. Burdeau Traité de Science/Politique Tome III, 2. Aufl. (Paris 1968). Der 
deren Ursachen man selbst gesetzt hat: Wenn man Idealen wie einer ins Unendliche ständig steigenden Produktivität der Wirtschaft und einem sich entsprechend ausbreitenden hohen Genuß für alle huldigt ${ }^{37}$, dann muß man sich mit der Möglichkeit befreunden, daß der klassische Verfassungsstaat etwas Derartiges nicht mehr zu versprechen und zu verfassen vermag, daß also ohne grundlegende Verfassungsänderungen eine Anarchie elementarer und gigantischer Kräfte, sowohl der Natur wie der Gesellschaft unvermeidlich wird. Wenn man sich der Einsicht in solche Zusammenhänge verweigert, dann rettet man nichts, gibt vielmehr lediglich den Weg frei für die Machtergreifung von Militärs, eine Fehlentwicklung, für die die "Freiheitsfreunde" sich der Verantwortung nicht dadurch entledigen können, daß sie sie verurteilen.

Bb) Die zweite Variante einer Totalrevision, die praktisch auf eine grundlegend neue Verfassung hinaus will, knüpft nicht an eine ganz andere Zukunft an, sondern will ohne solche Nötigung von sich aus eine andere Zukunft herbeiführen oder wenigstens einleiten. Ein solcher Entschluß braucht nicht unbedingt aus einer Ideologie zu entspringen - eine Möglichkeit, die gerade auch an dieser Stelle unbedingt $\mathrm{zu}$ verwerfen ist. $\mathrm{Zu}$ denken ist vielmehr an eine Situation, deren Eigenart darin besteht, daß sie das Empfinden der mars major oder der pars sanior des Volkes nicht mehr befriedigt, wobei insbesondere an das sittliche Empfinden gedacht ist. Eine solche Situation scheint heute insofern im Westen gegeben und im Osten sich abzuzeichnen als das ökonomische Element sowohl im einzelnen wie im ganzen überall vorherrscht, und zwar zu Lasten aller andersartigen Elemente wie Kultur, Politik und Sittlichkeit ${ }^{38}$. Zwar muß man durchaus damit rechnen, daß der Mensch bereit ist, vom Brot allein zu leben vorausgesetzt natürlich, daß dieses Brot ein reichliches und müheloses ist, also nicht etwa "Im Schweiße des Angesichts erworben und gegessen werden muß“. Aber das Gegenteil ist nicht mit derjenigen Sicherheit auszuschließen, die es einem vorausschauenden Verfassunggeber gestatten würde, eine solche Möglichkeit als undenkbar und daher als unbeachtlich beiseite zu schieben.

Käme er demgemäß zu dem Ergebnis, daß mit der zweiten Möglichkeit zu rechnen sei, der Mensch also das bloß ökonomische und genießende "Glück" nicht mehr als Erfüllung seines Menschentums ansehen könnte, dann würde er sich nach der hier vertretenen Auffassung in einer solchen Situation zu entschließen haben, den Eintritt dieser Möglichkeit wenigstens zu „kanalisieren“ und dieserhalb wieder in Kauf zu nehmen, daß z. B. diejenigen Freiheitsrechte, die kommerziell entartet sind, wieder auf ihre höhere Bedeutung zurückgeführt würden.

\section{Grundhaltung und Grundstimmung}

Der "Situation“ als der zeitlichen und räumlichen Umwelt, innerhalb deren und unter der der Verfassunggeber $\mathrm{zu}$ überlegen und $\mathrm{zu}$ wirken hat, entsprechen als Topos eine Geistesverfassung und Gestimmtheit —, innere Gegebenheiten, von

Wahlen-Bericht S. 17 beschränkt sich zu alledem auf folgende Bemerkung: „Zum Beispiel wird man froh sein müssen, wenn ein gewisses Gleichgewicht zwischen Parlament und Regierung die frühere vorherrschaft des Parlaments ablöst und die jetzige Vorherrschaft der Regierung sich nicht noch mehr ausprägt.*

37 Wenn man z. B. weitere und einschneidende Verkürzungen der Arbeitszeit, Verdoppelung und Verdreifachung des Urlaubs usw. und steigenden. Wohlstand fordert, dann sollte doch die Frage wenigstens aufgeworfen werden, ob sich dies alles staatlich und konstitutionell ohne Diktatur verwirklichen läßt.

38 Vgl. hierzu näher Herbert Krüger, Paritätische Mitbestimmung - Unternehmensverfassung - Mitbestimmung der Allgemeinheit (Düsseldorf 1973), S. $12 \mathrm{ff}$. 
denen ohne weiteres soviel auszumachen ist, daß sie von einer Eigenart sein müssen, die eine gute Verfassung verspricht. Von solchen Voraussetzungen soll im folgenden die Rede $\operatorname{sein}^{39}$.

\section{Die Grundhaltung}

a) Der Wahlen-Bericht bildet hierzu zwei Alternativen. Einmal mag der Verfassunggeber sich von "Ideen" oder gar einer "Tragenden Idee" leiten lassen. Zum zweiten mag er von den Problemen ausgehen, die die Wirklichkeit stellt. Der Bericht entscheidet sich für eine Kombination von beidem, mit Übergewicht der zweiten Alternative ${ }^{40}$. „Eine glaubwürdige Verfassungsrevision wird auf hohe Gedanken nicht verzichten: Aber ihre Fragen (vom Bericht gesperrt) holt sie aus der staatlichen Realität." Angesichts der in der BRD vorwaltenden Neigung, die Antwort auf Verfassungsfragen aus hohen und höchsten Prinzipien zu deduzieren, lassen solche Sätze des Berichts aufhorchen, und man ist daher erst recht begierig, zu erfahren, wie diese Realität sich in den Augen der Arbeitsgruppe darstellt. Als erstes ist diese Gruppe offenbar der Meinung, daß die Verfassung von 1874 dieser Realität nicht mehr entspricht und genügt: „Denn da liegt ja die wundeste Stelle der heutigen Verfassungen: daß sie in größerer Zahl untergeordnete, gleichgültige und entfallene Fragen regeln, daß sie an den politisch aufwühlenden Sachproblemen in deren Neuartigkeit vorbeisehen und so ein Leben fristen, das nicht mehr das Zentrum der politischen Gestaltungsaufgaben berührt." Das sind harte Worte, und es setzt danach nicht mehr in Erstaunen, wenn der Bericht weiter feststellt, daß die Hochschätzung, die das 19. Jahrhundert den Verfassungen entgegengebracht habe, einer ziemlichen Indifferenz gewichen sei ${ }^{41}$.

Als zweite Beobachtung, die die Arbeitsgruppe von der, von ihr zugrundegelegten Realität getroffen hat, besteht in "einem wachsenden Freiheitsanspruch": Er wird eingehend gewürdigt. Dann wird der Föderalismus als eine Strömung gewertet, der nicht allzuviel Kraft mehr innewohne. Als offene Elementarprobleme der Totalrevision werden schließlich vorgeführt etwa der Pluralismus der Gesellschaft, die "Mündigkeit" des Menschen und schließlich eine Reihe neuer Sachverhalte, die um die Stichworte „Leistungsstaat“, „Partei- und Verbandswesen“ und schließlich die Erscheinungsformen der unmittelbaren Demokratie kreisen.

b) Der gerade in der Gegenwart höchst billigens- und beherzigenswerte Entschluß eines Verfassunggebers, nicht aus einer Ideologie zu deduzieren und die Ergebnisse solcher Deduktion einer womöglich widerstrebenden Wirklichkeit aufzuzwingen ${ }^{42}$, sondern von der Wirklichkeit auszugehen, bedarf der Erläuterung dahin, worin die Kunst der Verfassunggebung die ihr aufgegebene Wirklichkeit $\mathrm{zu}$ suchen hat, so wie das Verfassungsrecht sich klar darüber sein muß, welche

39 Angesichts der Unfaßlichkeit dieser Gegenstände muß ihre Erkenntnis notgedrungen noch subjektiver ausfallen, als dies im allgemeinen für wissenschaftliche Erkenntnisse gilt.

40 S. 43.

41 Als Beispiel, daß eine solche Prüfung der Wirklichkeit nicht einmal erwogen wird, vielmehr alles vorgefaßte Meinung ist, sei hingewiesen etwa auf Juan J. Linz, The Future of an Authoritarian Situation or the Institutionalization of an Authoritarian Regime: The Case of Brezil, in: A. Stepan (ed.) Authoritarian Brazil (Yale UP 1973), S. $233 \mathrm{ff}$. 
Wirklichkeit die ihm zugeordnete, also die spezifische „Verfassungswirklichkeit“ ist. Hierzu wird für die Verfassunggebung ohne weiteres Zustimmung finden die These, daß Symptome, ja sogar eine Reihe von Symptomen nicht dieWirklichkeit sind, mit der es der Verfassunggeber $\mathrm{zu}$ tun hat, daß sie aber von unschätzbarer Bedeutung für ihn sind deswegen, weil sie auf diese Wirklichkeit und ihre Krankheit aufmerksam machen („Menetekel“!). Diese Erkenntnis belegt im Augenblick geradezu klassisch die Angelegenheit Nixon: Für die Sicht des Verfassunggebers ist hier nicht erheblich die Person, sondern die Umstände, die es geduldet oder geradezu unvermeidlich gemacht haben, daß ein Mensch solchen Schlages Präsident werden konnte. Das Symptom „Nixon“ hätte also alle Veranlassung gegeben, das Verfahren einmal unter die Lupe zu nehmen, mittels dessen das Volk der Vereinigten Staaten seinen Präsidenten kreiert. Man braucht sich nur die einzelnen Abschnitte dieses Verfahrens zu vergegenwärtigen, insbesondere die dem Kandidaten auferlegten unmenschlichen physischen Anstrengungen, um zu dem Ergebnis zu gelangen, daß es nur „Bärennaturen“, also Typen von der Art Nixons möglich ist, ein solches Verfahren durchzustehen und zu finanzieren! Wenn man derartige Typen möglichst schon von der Kandidatur fernhalten und angemessenere gewinnen will, dann muß man ein Verfahren ändern, das im Grunde nur bedenkenlose und hartgesottene Figuren als Kandidaten zuläßt. Der Fall Nixon wäre also nicht nur ein Fall für das Parlament, die Offentlichkeit und vielleicht die Gerichte gewesen, er war und ist vor allem ein Fall für den Verfassunggeber. Dieser aber hat sich, soweit ersichtlich, nicht einmal zu einschlägigen Überlegungen veranlaßt gefühlt. Man hat sich damit begnügt, an den Symptomen herumzukurieren, Nixon in die Wüste zu schicken und sich der hierdurch bewirkten Katharsis zu erfreuen. Fragt man nach den Ursachen solcher Enthaltung, dann mag man auf eine Wohlstandsgesellschaft stoßen, die ihre Unlust, sich mit ernsthaften Dingen zu beschäftigen oder gar radikale Entscheidungen zu treffen, durch die Lust kompensiert, die eine maßlose moralische Entrüstung offenbar zu erregen vermag - die Verschiebung eines Problems aus dem Zusammenhang "Fehler" — "Abhilfe“ auf die Ebene von "Schuld" und "Sühne“, die nicht nur weniger anstrengt, sondern zudem erbaut ${ }^{43}$.

c) Wenn somit, um nur das mindeste zu sagen, der sich mit der Wirklichkeit auseinandersetzende Verfassunggeber sehr viel tiefer dringen muß als nur bis zur Oberfläche der Symptome, dann stellt sich die Frage, wie es mit den Hilfen steht, ohne die der Verfassunggeber sich Kenntnis und Deutung seiner Wirklichkeit bis in ihre tiefsten und wesentlichsten Schichten schwerlich zu verschaffen vermag, was also die politischen Ideologien hierfür anzubieten haben. Die Antwort fällt nicht zu scharf aus, wenn sie lautet: Nichts oder jedenfalls so gut wie nichts.

Das gilt als erstes für den Neo-Marxismus ${ }^{44}$. Wenn er sich nicht von vornherein auf Begrifflichkeit und Beschäftigung allein mit seinen Begriffen beschränkt $t^{45}$,

43 Das entsprechende gilt übrigens auch und gerade für die Volksvertretungen: Auch hier beschäftigt sich die Offentlichkeit vorwiegend mit der Aufdeckung von Skandalen oder gar globaler moralischer Verdammung, während man sich kaum jemals fragt, wie man es bewirken könne, daß wirklich nur die Besten kandidieren und nur die Allerbesten gewählt werden.

44 Vgl. hierzu etwa: Urs Jaeggi, Kapital und Arbeit in der Bundesrepublik - Elemente einer gesellschaftlichen Analyse (Frankfurt am Main 1968, 1973), Ernest Mandel, Der Spätkapitalismus (Frankfure am Main 1972).

45 Vgl. etwa Jürgen Habermas, Legitimationsprobleme im Spätkapitalismus (Frankfurt am Main 1973). 
dann gewinnt er sein gegenwärtiges Bild der Wirklichkeit in erster Linie dadurch, daß er aus den Lehren Marxens und Engels das herausfällt, was diese Autoren ihren Überlegungen als Wirklichkeit zugrundegelegt haben. Ein solches Vorgehen ist nicht ohne die Annahme denkbar, daß Marx diese Wirklichkeit für alle Zeiten und Räume endgültig erfaßt und bestimmt hat und daß es daher eines neuerlichen, oder gar ständig neuer Blicke auf die Wirklichkeit nicht bedarf, ja daß sie eher schädlich sind, weil sie die Erkenntnis Marxens nur trüben könnten. Auf diese Weise wird „Klassenkampf“, und zwar der auf zwei Gruppen reduzierte, totale und letzte Klassenkampf, in dem sich "Ausbeuter" und "Ausgebeutete“ gegenüberstehen, zur Wirklichkeit schlechthin. Sie bedarf keiner Erforschung mehr, man kann vielmehr ebenso wie es die "Ausbeuter" zu tun vorziehen (vgl. den oben gedeuteten Fall Nixon) sogleich zu der, Anstrengungslosigkeit wie Erbaulichkeit vereinigenden moralischen Entrüstung übergehen. Damit hat man sich den Blick auf die aktuelle und wesentliche Wirklichkeit endgültig verstellt. $\mathrm{Da}$ es sich in der Tat so verhält, zeigt der Versuch einer Verdeutlichung, wie etwa im Ergebnis die "revolutionäre“ Veränderung aussehen würde, auf die die Neo-Marxisten ausgehen. Da es eine fiktive Wirklichkeit ist, die sie ablösen wollen, wird die wirkliche Wirklichkeit in ihrer Revolution nicht getroffen werden: Die „Industrielle Gesellschaft" mit ihren Idealen, Strukturen und Methoden würde unverändert aus dieser Veränderung hervorgehen; es würden dieselben Leute oder jedenfalls derselbe Typ von Leuten die Unternehmen "managen“, und der Arbeiter würde nach wie vor in gleicher Weise etwa die Presse bedienen, mit der er tagaus tagein einen bestimmten Kotflügel für ein bestimmtes Auto herstellt ${ }^{46}$.

Auch die liberal-soziale und sozial-liberale Weltanschauung fußt auf einem Bild der Wirklichkeit, das im günstigsten Falle der Vergangenheit angehört ${ }^{47}$ und daher im Ergebnis ebenso unwirklich ist wie die Wirklichkeitsvorstellung des Neo-Marxismus. Wie sich aus der Anti-Kartell-Gesetzgebung als eine Art von für diese Seh- und Denkweise vor allem bezeichnenden Leitfossil erschließen läßt, setzt sich für sie die Gesellschaft immer noch aus Einzelgebilden zusammen, die sich durchweg antagonistisch zueinander verhalten (vor allem: Wettbewerb!) und die mittels solchen egozentrischen Antagonismus selbsttätig das im einzelnen und im ganzen richtige Ergebnis hervorbringen. Verborgen oder geleugnet bleibt bei allem, daß die Industriegesellschaft ganz anders, vor allem kollektiv strukturiert und arbeitet (Allgemeine Geschäftsbedingungen; Tarifvertrag usw. usw.) und daß sie vor allem makroökonomisch nicht mehr aleatorisch, sondern rational operiert. Wie der Neo-Marxismus beschränkt sich auch die liberal-soziale Ideologie nicht damit, die wirkliche Wirklichkeit und ihre Tendenzen zu verkennen, sondern versucht ebenfalls, sie nach dem Bilde ihrer Ideologie zu ändern anstatt die Ideologie im Blick auf die Wirklichkeit zu verändern.

Der Konservativismus hat zwar im 19. Jahrhundert Autoren wie G. F. W. Hegel oder Lorenz von Stein hervorgebracht, die mit einer Intutition ohnegleichen die

\footnotetext{
46 In diesem Zusammenhang ist auch jene Auffassung zu erwähnen, die im Streik eine Art von deus ex machina sieht, der allen Ubeln ein für allemal auf einen Schlag abhelfen wird: Wie Gewalt und Kampf sich mit einer durch und durch rationellen Gesellschaft und vor allem einer solchen Produktionsweise vertragen, wird offenbar nicht gefragt. Romantik ist alles.

47 Dies hat G. Burdeau, Traité de Science Politique 2. Aufl. VI/1 (Paris 1971): „Les Assises intellectuelles et Sociales de l'Etat Libéral“" herausgearbeitet.
} 
Wirklichkeit ihrer Zeit erkannt und in Gedanken gefaßt haben. In der Gegenwart erweist er sich auch an dieser Stelle als nicht vorhanden ${ }^{48}$

Insgesamt ergibt dieser tour d'horizon: Diejenigen Helfer, die den Verfassunggeber vor allem bei der Gewinnung eines Bildes der Wirklichkeit zu unterstützen hätten, lassen ihn im Stich, wenn sie ihn nicht sogar irreführen.

d) Ohne hiernach viel mehr zu wissen, als daß der Verfassunggeber sich mit der von $\mathrm{ihm} \mathrm{zu}$ verfassenden Wirklichkeit vertraut $\mathrm{zu}$ machen und mit ihr auseinanderzusetzen habe, müssen wir nunmehr versuchen, zu klären, wie etwa eine solche Auseinandersetzung auszusehen hätte.

Wenn der Staat der Neuzeit zu begreifen ist als die festere Einheit und die bessere Gestalt, in die sich ein Volk unablässig bringt, um sich in den es bedrohenden oder angehenden, äußeren und inneren, heute vor allem selbstgeschaffenen (Wohlstandsgesellschaft; Luftverschmutzung usw. usw.) „Lagen“ erfolgreich behaupten zu können ${ }^{49}$, dann ist der Verfassunggeber vor allem gehalten, seine Tätigkeit als Auseinandersetzung mit solchen "Lagen“ zu begreifen, indem er Feststellung und Bewältigung solcher Lagen programmiert.

Dies mag im einzelnen etwa besagen:

aa) Die These, daß der Verfassunggeber nicht von einer Ideologie, sondern von seiner Wirklichkeit auszugehen habe, wird hierdurch endgültig bestätigt.

bb) Die erste Aufgabe eines, einen Modernen Staat verfassenden Verfassunggebers stellt sich hiernach dahin, daß er die für ihn wesentlichen "Lagen“ erkennen und würdigen soll, mit denen es dieser Staat zu tun hat. Es braucht kaum hervorgehoben $z u$ werden, daß dies die schwierigste und wichtigste Aufgabe des Verfassunggebers ist, und daß mit der Qualität ihrer Erledigung das Entscheidende über die Qualität der Verfassung gesagt ist.

Dies sei zunächst an der „Auswärtigen Lage“ erläutert, obwohl man sich heute nicht mehr darüber einig ist, ob der Auswärtigen oder der Inneren Lage der Primat zukommt. Vom Wahlen-Bericht ist als erstes zu rühmen, daß er den "Lagen" seine Aufmerksamkeit gewidmet hat ${ }^{50}$. Unter ihnen wird auch der "Auswärtigen Lage“ Beachtung geschenkt. Hierzu stellt er fest, daß die äußere Bedrohung eines Staates heute weniger eine militärische als eine psychologische ist, der Verfassunggeber daher den Erfordernissen der „Psychologischen Landesverteidigung" (625) Rechnung zu tragen hat, indem er auf Stärkung der seelischen Abwehrkraft (620) bedacht und notfalls bereit ist, dieserhalb einen Abbau an der Unmittelbaren Demokratie in Kauf zu nehmen (656)

Es mag der Veranschaulichung dieses Punktes dienen, wenn man etwa näher auf die „Psychologische Landesverteidigung“ eingeht. Sie verdankt ihre Aktualität der Erscheinung des „Psychologischen Krieges ${ }^{52 “}$ : Je weniger der militärische Krieg jedenfalls großen Stils noch in Betracht kommt, um so mehr tritt die Psychologische Kriegführung in den Vordergrund. Wenn für diesen Krieg jeder Bürger geborener Verteidiger seines Vaterlandes in noch viel weiterem Umfang und mit noch

48 Wenn man ihn mit „konservativ“ richtig einordnet, dann wäre hier Ernst Forsthoff vor allem mit „Der Staat der Industriegesellschaft" (München 1971) zu nennen.

49 Dies ist der Tenor meiner "Allgemeinen Staatslehre“ (Stuttgart 1964, 1966); zu geistesgeschichtlichen Einordnungen vgl. diese Zeitschrift 7 VRU (1974), S. $55 \mathrm{ff.:}$ Zur Bestimmung des geistesgeschichtlichen Standorts von Staatstheorien.

50 S. $618 \mathrm{ff} .:$ "Die heutigen Bedrohungen eines Staates; vgl. auch S. 703.

$51 \mathrm{Vgl}$. auch "a. a. O. S. 35: „Zu fordern ist schließlich, daß eine neue, total revidierte Verfassung in ihrer Form Einfügungen der Schweiz in europäische Zusammenschlüsse und deren Grundnormen erleichtert, zumindest aber nicht erschwert."

52 Vgl. hierzu meinen Beitrag zur Festschrift Werner Weber (Berlin 1974): „Verfassunggebung in Hinblick auf die Auswärtige Lage." 
höheren Ansprüchen ist, als im militärischen Krieg, weil hier auch der militärisch „Untaugliche“ angegriffen und der Abwehr fähig ist, dann handelt es sich allerdings um eine "Lage“, die der Verfassunggeber zu erkennen und zu bewältigen hat. Diese Lage und ihre Bewältigung differenziert sich zwischen Ost und West dadurch, daß man sich im Osten für eine vor allem auch nach außen "geschlossene" Gesellschaft entschieden hat, nicht zuletzt um das moralische "Gift" des Kapitalismus-Imperialismus von den Bürgern von vornherein fernzuhalten, während der Westen auch in dieser Beziehung an einer "Offenen“ Gesellschaft festhält ${ }^{53}$. Gleich aus welchen Gründen ein westlicher Verfassunggeber dies tut, auf jeden Fall darf er sich auch an dieser Stelle nicht darauf verlassen, daß irgendein Automatismus diesen Nachteil selbsttätig ausgleichen werde, er muß vielmehr selbst Überlegungen anstellen und Entscheidungen treffen, die die Waffengleichheit wiederherstellen. Er muß sich also vergewissern, wie es in Köpfen und Herzen seiner Bürger aussieht, ob sie etwa in den amtlichen Idealen, Werten usw. eigene Ideale, Werte usw. sehen, ob diese ihrer Bejahung eine platonische bleibt oder ob sie bereit sind, für sie und das durch sie bestimmte Gemeinwesen „auf die Barrikaden" zu gehen usw. usw. Da diese Diagnose gewiß nicht sogleich voll befriedigend ausfallen wird, wird er zunächst nach den Ursachen des Unterschusses zu fragen haben, insbesondere also ob die Massenmedien der ihnen gerade an dieser Stelle obliegenden Aufgabe genügen usw. usw. Nicht zuletzt wird er zu erwägen haben, wie es mit der Verteidigungsbereitschaft einer Wohlstandsgesellschaft überhaupt bestellt, insbesondere ob ihr nicht eine Gleichgültigkeit gegen das nationale Schicksal überhaupt eingeboren ist ${ }^{54}$.

Die Innere Lage bedarf nicht minder als die Auswärtige der eindringenden Diagnose des Verfassunggebers. Hierher gehört als das allgemeinste, daß er sich ein Bild von den Menschen macht, mit denen er es zu tun hat und die der Verfassung Lebendigkeit und Wirksamkeit vermitteln sollen. Im besonderen wird er $\mathrm{zu}$ überlegen haben, welcher Verfassung eine Überfluß- und Verbrauchsgesellschaft bedarf, damit sie das vorfindet und erhält, worauf ihr Begehren gerichtet ist, vor allem also mehr Güter, mehr Freizeit und vor allem mehr Unterhaltung. Eine solche Überlegung wird mit dem Staatstyp zu beginnen haben, der unter solchen Umständen nicht nach seiner Idealität, sondern nach seiner Brauchbarkeit $\mathrm{zu}$ bestimmen ist ${ }^{55} \mathbf{5 6}$.

\section{Von der Stimmung, in der eine Verfassung gegeben wird}

Die Notwendigkeit, sich mit der, den Anforderungen an eine Verfassung entsprechenden Gestimmtheit des Verfassunggebers zu beschäftigen, mag exemplarisch verdeutlicht werden durch die Überlegung, daß der Tag der Verfassunggebung dazu prädestiniert ist, nationaler Feiertag zu werden. Eine Verfassung aber kann

$53 \mathrm{Vgl}$. hierzu das Bundesverfassungsgericht vom 25. April 1972, in: BVerfGE 33, S. $52 \mathrm{ff}$.

54 Der einzige, der diese Frage aufgeworfen hat, scheint mir Karl de Schweinitz, Industrialization and Democracy (Free Fress of Glencoe 1964) gewesen zu sein; neuerdings auch Gr. Burdeau im VIII. Band seines oben erwähnten Traité. . . (Paris 1974).

55 Einige weitere Beispiele von Erwägungen, die Verfassunggeber unter dem Blickwinkel der Auswärtigen Lage angestellt haben, finden sich bei J. S. Coleman und C. G. Rossberg, Political Parties and National Integration in Tropical Africa (California UP 1970), S. 26.

$56 \mathrm{Vgl}$. hierzu auch Christian Tomuschat, Güterverteilung als rechtliches Problem, in: Der Staat (1973), S. 433 ff., S. 465: Verknappung macht Güterverteilung durch den Staat notwendig. Hierauf ist das Grundgesetz als liberal geprägte Verfassung nur wenig gerüstet. 
man naturgemäß nur dann wahrhaft feiern, wenn die Erinnerung an diesen Tag eine Feierlichkeit im Bewußtsein der Nation auslöst. Eine solche Stimmung wiederum kann man nur erwarten, wenn die Verfassung als solche, vor allem aber der Vorgang ihrer Schöpfung als etwas Feiernswertes empfunden werden kann. Ein Verfassunggeber muß sich dazu nicht zuletzt darüber vergewissern, wie es mit dem Geist bestellt ist, von dem gestimmt er die Verfassung schöpft. Wenn sich gewiß auch ein guter Geist nicht erzwingen läßt, so läßt sich doch wohl verhindern, daß ein schlechter Geist Herrschaft über die Verfassunggebung gewinnt ${ }^{57}$.

Es versteht sich von selbst, daß die Feiernswürdigkeit einer Verfassung zwar ein wichtiger, aber keineswegs der einzige Grund ist, der dafür spricht, nach der Gestimmtheit des Verfassunggebers zu fragen. Es kommt hier vor allem hinzu die Überlegung, die sich im Zusammenhang mit der materiellen Normativität einer Verfassung aufdrängt ${ }^{58}$ : Soll sie überhaupt nicht der Abklatsch der Wirklichkeit, vor allem aber nicht einem Tiefpunkt der nationalen Existenz zugänglich sein, soll sie vielmehr das „Bessere Ich“ eines Volkes, das sich im Staat repräsentiert, zu Präsenz, Aktivität und Durchsetzung auch und gerade in „schlechten Tagen“ bringen, dann folgt hieraus, daß die Verfassunggebung selbst einen Höhepunkt des Nationalen Lebens darstellen, also regelmäßig auf einem solchen Höhepunkt erfolgen muß. "Höhepunkt" meint insoweit „Hochstimmung", ein Hochgefühl der Kreativität oder wie immer man eine solche Geistes- und Seelenverfassung ansprechen mag. Etwas Derartiges mag wiederum aus der Überzeugung entspringen, daß man eine Revolution verfaßt, die der Weltgeschichte eine neue Wendung gibt wie etwa die amerikanische, die französische oder die russische Revolution ${ }^{59}$.

Es liegt auf der Hand, daß es sich bei alledem um die subjektive Seite dessen handelt, was früher als "Situation“ zu erfassen und zu beschreiben unternommen worden ist. Der ideale Fall ist dann gegeben, wenn eine Verfassung in einer "Sternstunde" hervorgetreten ist, die einen Höhepunkt sowohl in der nationalen Geschichte wie in der Gestimmtheit der Nation darstellt. Damit ist nicht gesagt, $\mathrm{daß}$ in einer nationalen Katastrophe eine solche Hochstimmung undenkbar wäre: Die Reaktion kann vielmehr gerade in einem solchen Falle ein Wille zur Erneuerung und Neugeburt sein, der nicht mindere Werke zu verschaffen vermag als ein Sieg.

Diejenige Gestimmtheit, auf die es hiernach ankommt, läßt sich nicht, wie schon angedeutet, künstlich „machen“. Die Lehre von der Kunst der Verfassunggebung vermag aber sehr wohl zu erforschen, was einer solchen Gestimmtheit zuwider ist, was also „verstimmend“ wirken kann. In diesem Zusammenhang ist immer noch an erster Stelle zu nennen jene Ängstlichkeit, jene Kleingeistigkeit oder wie immer man eine solche Geistesverfassung nennen mag, die jeder größeren Entscheidung schon deswegen, weil sie Entscheidung ist, ein tiefeingewurzeltes und offenbar unausrottbares Mißtrauen entgegenbringt. Was schon für die Entscheidung gilt, gilt naturgemäß erst recht für Macht und Herrschaft als Voraussetzungen rechtzeitiger und wirksamer Entscheidungen: Es ist nicht nötig, diejenigen Stimmen wiederzugeben, die sie als schlechthin böse bezeichnen, es genügt der Hinweis darauf, daß solche Mißgestimmtheit heute der Forderung nach allgemeiner „De-

\footnotetext{
57 Es mag sein, daß Gottesdienste zu Beginn einer Verfassunggebenden Versammlung oder die Anrufung Gottes im Verfassungstext einen solchen Geist beschwören sollen.

58 Vgl. oben Anm. 23.

$59 \mathrm{Es}$ sind dies die einzigen Revolutionen, die P. Nehru beeindruckt haben; vgl. M. Brecher, Nehru A Political Biography - (London, Oxford UP 1959) S. 329
} 
mokratisierung" zugrundeliegt, insofern sie allenthalben die radikale Beseitigung von Macht und Herrschaft verlangt. Einer der Väter einer solchen Einstellung ist Karl von Rotteck (1775-1840). Für ihn hat eine Verfassung zwei Aufgaben: Erstens soll sie künstliche Organe in einem einmaligen Akt einrichten; zweitens soll sie diesen Organen die Schranken bestimmen, um ihnen den Mißbrauch der Macht zu verwehren. Die zweite Aufgabe ist die eigentlich fortdauernde. Das Zentralproblem der Verfassung ist also die Beschränkung der Macht durch ihre Struktur; diese Frage bildet den Inhalt des Verfassungsgesetzes ${ }^{60}$. Es scheint insgesamt so, daß diese Gestimmtheit die Gefahren eher gefördert hat, gegen die sie sich wendet sie hat insbesondere die Bildung apokrypher Macht begünstigt. Auf jeden Fall hat sie das Problem umgangen, das einer Verfassung immer, vor allem aber in einer Wohlstandsgesellschaft gestellt ist: Eine Mächtigkeit des Gemeinwesens zu konstituieren, die ebenso fähig ist, die erforderlichen Entscheidungen zu treffen und durchzusetzen, wie diese Fähigkeit allein durch die Notwendigkeit solcher Entscheidungen bestimmt wird: Sie macht es sich leicht, indem sie sich darauf beschränkt, die irdischen Voraussetzungen von Handlungs- und Leistungsfähigkeit des Gemeinwesens zu verdammen und ihm soweit wie möglich vorzuenthalten ${ }^{61}$.

Eine andere, in unserer Zeit sich bemerkbar machende Fehlgestimmtheit der Verfassunggebung ist Optimismus, der weniger das Gegenteil des eben umrissenen Mißtrauens als eher dessen Voraussetzung ist: Meint der Optimist, ein Gemeinwesen könne weitestgehend ohne Herrschaft und Macht auskommen, dann wird er allen Bemühungen, es dennoch mit beiden in dem erforderlichen Maße auszustatten, mit Mißtrauen begegnen. Ein solcher Optimismus äußert sich vor allem in der Wahl des Ausgangspunktes: Er wird die „Lagen und ihre Ernsthaftigkeit zugunsten einer Ideologie vernachlässigen, die eine heitere Welt verheißt, ohne daß es dieserhalb bedeutenderer Anstrengungen bedürfte. So werden denn Grundrechte in immer größerer Zahl und von immer erheblicherem Gewicht eingeräumt, ohne daß man auch nur fragte, wie der Mensch aussieht, dem man solche Freiheiten sichert, oder sich gar Gedanken darüber machte, wie er sie füglich gebrauchen sollte, damit sich das programmierte Gesamtergebnis mit einem Mindestmaß von Wahrscheinlichkeit einstellt. Bekanntlich ist es die Marktwirtschaft, die vor allem einem solchen Optimismus huldigt: Wenn der Staat nicht stört, sorgt hier die Natur automatisch bestmöglich dafür, daß die Konfrontation der Egoismen im Wettbewerb die für die einzelnen und die Gesamtheit erwünschten und erforderlichen Ergebnisse hervorbringt. Der Primat der Ókonomie hat bewirkt, daß dieser Optimismus von der Wirtschaft auf Gesellschaft, Staat und Politik übergegriffen hat.

Mit „Mißtrauen“ und „Optimismus“ ist die Zahl der möglichen Mißgestimmtheiten des Verfassunggebers naturgemäß keineswegs erschöpft. Der recht gestimmte, also "hochgemute" Verfassunggeber wird sich solcher Verstimmtheiten nicht nur zu

60 Nach E. Schmidt-Aßmann, Der Verfassungsbegriff in der deutschen Staatslehre der Aufklärung und des Historismus (Berlin 1967), S. 108. Mir scheint, daß sich heute eine solche Gestimmtheit etwa bei Werner Kaegi, Die Verfassung als rechtliche Grundordnung des Staates (Zürich 1945) findet. Wenn der Wahlen-Bericht S. 14 meint: „Die Verfassung führt dazu, daß die Macht des Staates beschränkt und kanalisiert werden kann", dann wird nicht recht deutlich, ob hiermit eine fremde Meinung (sc. des Konstitutionalismus) oder eine eigene Auffassung wiedergegeben wird.

61 Typisch hierfür pflegt die Behandlung des Verordnungsrechts der Exekutive zu sein, und zwar gerade in parlamentarischen Systemen. Aber auch hier zeigt es sich, daß die Natur "semper revertit “; vgl. J. Souberrol, Les Décrets-Lois sous la Quatrième République (Paris 1955). In "der BRD ist Art. 80 ein beredtes Zeugnis solcher Fehlgestimmtheit: An das Gesetz, dessen der Verordnunggeber als Ermächtigung bedarf, sind solche Anforderungen gestellt, daß ihnen der Gesetzgeber genau genommen nur dadurch genügen könnte, daß er die Verordnung selbst erläßt. 
erwehren, er wird vielmehr den Versuch unternehmen müssen, auch die von ihm geschaffene Verfassung vor Durchdringung durch solche Fehlgestimmtheiten zu bewahren. Man wird ohne weiteres davon ausgehen können, daß dies in allzu vielen Fällen nicht geschehen ist, dort aber, wo dergleichen bedacht worden ist, Theorie und Praxis sich dennoch nicht haben davon abhalten lassen, die Verfassung und die Bürger in eine solche Mißstimmung zu versetzen. Davon wird später noch einmal die Rede sein ${ }^{62}$.

\section{Die essentielle Thematik einer modernen Verfassung}

Das zunächst wichtigste dieses Dritten Kapitels einer Untersuchung, die der Kunst der Verfassunggebung gewidmet ist, hat man darin zu sehen, daß es einen Gegenstand behandelt, dem der Verfassunggeber seine Aufmerksamkeit und seine Überlegungen zu schenken hat: Wie auch der Gesetzgeber, so hat vor allem er sich an erster Stelle darüber klarzuwerden, welche Materien in eine Verfassung aufgenommen und welche umgekehrt aus ihr ferngehalten werden müssen.

Insbesondere ergibt sich eine solche Notwendigkeit erstens aus dem Erfordernis, daß der Verfassungstext nur Verfassungssätze von Verfassungsrang, also nur „materielles" Verfassungsrecht, dieses aber vollständig enthält ${ }^{63}$. Zweitens muß Klarheit über die essentielle Verfassungsthematik dort bestehen, wo wie in der Schweiz eine Gesetzesinitiative des Volkes zwar in den Einzelstaaten, nicht jedoch im Gesamtstaat besteht, der lediglich eine Verfassungsinitiative kennt: Man wird also im Gesamtstaat versuchen, eine Regelung, die allenfalls der Form des Gesetzes würdig ist, als Verfassungsinitiative zu lancieren, um überhaupt zu einer Volksinitiative zu gelangen. Will man solcher Verfassungsumgehung wehren, dann muß man imstande sein, Regelungen ohne Verfassungsrang als solche zu definieren, um sie zurückweisen zu können. Richtigkeit und Notwendigkeit des Vorhabens, die essentielle Thematik einer modernen Verfassung festzustellen, ergibt schließlich eine Beobachtung, die Norbert Wimmer ${ }^{64}$ gemacht hat: "Die modernen Verfassungen haben freilich den Sinn für die Wesentlichkeit ihres Inhaltes weitgehend verloren“. Diese Beobachtung wird durch einen Blick auf die Theorie bestätigt. Der Wahlen-Bericht ${ }^{65}$ bemerkt hierzu: „Zur Frage der Form im weiteren Sinne dieses Wortes gehört, welche Regelungsbereiche die geschriebene Verfassung aufzunehmen hat. Eine feststehende Lehre hierüber ist nicht vorhanden ... (Vom Bericht gesperrt). Für die weitere Diskussion in der Schweiz dürfte sich der Regelungsbereich jedenfalls aus drei verschiedenartig begründeten Sektoren zusammensetzen:

a) Die Rechtslogik gebietet in erster Linie, Bestimmungen über den Gesetzgeber aufzunehmen, das heißt, ein Gesetzgebungsorgan zu schaffen und ihm in Gestalt von Kompetenzen den Auftrag zu geben, die weitere Rechtsordnung zu gestalten.

$62 \mathrm{Vgl}$. hierzu auch meinen Beitrag „Uber die Unterscheidung der Staatstypen nach ihrer Gestimmtheit“, in: Festschrift für Hermann Jahrreiss (Köln usw. 1964), S. $233 \mathrm{ff.}$

63 Nach Boris Meissner, Die Verfassungsentwicklung der Sowjetunion nach dem Tode Stalins, in Jahrbuch des Offentlichen Rechts der Gegenwart, Neue Folge 22, S. 103, S. 125 ff. sind nicht weniger als drei bedeutende Gruppen von Normen mit Verfassungsrang außerhalb der Verfassungs-Urkunde placiert, also lediglich "materielles Verfassungsrecht ${ }^{\star}$.

64 Materiales Verfassungsverständnis (Wien-New York 1971), S. 11.

65 A. a. O. (oben Anm. 3), S. 36. Nicht unerwähnt soll bleiben, daß der Wahlen-Bericht an der Möglichkeit materieller Vollständigkeit einer Verfassung zu zweifeln scheint; vgl. S. 22: Eine Verfassung kann nicht mehr selbst alle wichtigen Grundentscheidungen bringen. 
Fällt der Entscheid, einen Bundesstaat einzurichten ... so fordert die Logik, Elemente der Aufgaben- und Kompetenzverteilung zwischen den Gemeinwesen beizubringen.

b) Den immer noch beachtlichen Postulaten des Konstitutionalismus ist sodann zu entnehmen, daß die Verfassung sich auszusprechen hat über:

- Grundentscheidungen bezüglich weiterer Staatsorgane sowie ihrer Funktionen und Zuständigkeiten (Volk, Exekutive, Judikative),

- die Festlegung, ob der Staat eine föderative Ordnung haben solle,

- das Grundverhältnis Bürger - Staat, namentlich die Grundrechte im klassischen Sinn und die Bürgerpflichten,

- Revisionsmöglichkeiten und -verfahren betreffend das Verfassungsrecht.

c) Schließlich wird die Verfassung auch Normen aufnehmen über Fragen, die aus den Bewertungen der Gegenwart als verfassungswürdig zu gelten haben, so

- Zielnormen staatlicher Betätigungen, und zwar in einem vielleicht größeren Ausmaß und in anderer Art als die geltende Bundesverfassung,

- Normen über die Staatsführung,

- Aufträge an den Gesetzgeber und weitere Staatsorgane, was in Kompetenzvorschriften eingeschlossen sein kann,

- neuartige Verhältnisbestimmungen betreffend den Menschen in seinen Beziehungen zu Staat und Gesellschaft, so vor allem Sozialrechte (soziale Grundrechte).“

Das essentielle Thema einer jeden modernen Verfassung haben wir schon früher beschrieben: Die Ordnung der Auseinandersetzung mit den Lagen, in denen sich ein Gemeinwesen vorfindet, gleich ob sie vorgegebene oder selbstgeschaffene sind. $\mathrm{Da}$ diese Lagen zahlreich und verschieden sein werden, läßt sich über sie unter dem Stichwort „Essentielle Thematik" einer Verfassung nicht mehr als das ausmachen, was eben gesagt worden ist. Darüber hinaus gibt es jedoch Gegenstände, die einer Verfassung allein dadurch aufgegeben sind, daß es sich um die Verfassung einer Gruppe, und zwar einer zu Staatlichkeit entschlossenen Gruppe handelt. Aus dem ersten ergibt sich die Notwendigkeit, Vorkehrungen für die Bildung und Festigung der Einheit dieser Gruppe zu treffen („Die Verfassung als Programm der Nationalen Integration“). Bedeutet der Entschluß zu Staatlichkeit Entscheidung für eine Selbststeigerung der Nation, um die Auseinandersetzung mit den Lagen mit Aussicht auf das bestmögliche Ergebnis führen zu können, dann muß eine entsprechende Verfassung einen solchen Entschluß konstitutionalisieren und seine Verwirklichung organisieren („Die Verfassung als Programm der Nationalen Repräsentation").

\section{Die Verfassung als Programm der Nationalen Integration ${ }^{66}$}

Eine jede Verfassung findet das Kollektiv, das sie verfaßt, nicht so vollständig und sicher kollektiviert vor, daß sie um eine solche nicht besorgt $\mathrm{zu}$ sein brauchte. Úberall ist vielmehr das Gegenteil mehr ${ }^{67}$ oder weniger der Fall, und demgemäß

66 Vgl. hierzu Herbert Krüger, Die Verfassung als Programm der Nationalen Integration, in: Festschrift für Friedrich Berber (München 1973), S. $24 \mathrm{ff}$.

67 Vgl. hierzu Heinrich Triepel, Der Streit um die Thronfolge im Fürstentum Lippe (Leipzig 1903), S. 13: "Und was das Reich anlangt, so kann gewiß für die politsche Einheit des deutschen Volkes nie genug getan werden." 
ist "Integration“ notwendiges Thema einer Verfassung eines jeden menschlichen Verbandes, vor allem aber des Staates. Verfassunggebung ist daher stets Konstituierung eines Verbandes oder eines Staates ${ }^{68}$, Verfassungsgeltung Erneuerung und Weiterbildung einer solchen Konstituiertheit. Es ist die sog., von Rudolf Smend ${ }^{60}$ begründete Integrationstheorie, die diesen Sinn der Verfassung aufgedeckt und dargelegt hat - , eine Lehre, deren Gehalt inzwischen Gemeingut der Wissenschaft geworden ist.

Kann somit kein Zweifel daran bestehen, daß die Einheit der Nation das erste und vornehmste Thema der Verfassung ist ${ }^{70}$, so stellt sich um so nachdrücklicher die Frage, wie der Verfassunggeber dieses Thema in der Verfassung zu behandeln habe. Von der Verfassung als Ganzheit ist in diesem Zusammenhang bereits die Rede gewesen ${ }^{71}$ : Noch vor ihrem Inhalt muß sie als einer Sternstunde der Nation entsprungene Schöpfung Bedeutung und Wert für die Bürger besitzen. Für die einzelnen Verfassungssätze muß unter dem Blickwinkel „Nationale Integration“ als erstes und vor allem festgehalten werden, daß sie von ganz anderer Art sind als etwa die Bestimmungen eines Bürgerlichen Gesetzbuches. Dies gilt vornehmlich von der Absicht, in der sie ihren Adressaten ansprechen: Dem Bürgerlichen Recht geht es nicht mehr als um ein äußeres Verhalten, auf die Gesinnung kommt es grundsätzlich nicht an, geschweige denn, daß solche Bestimmungen im Gewissen verpflichteten. Ganz anders verhält es sich mit den Verfassungsätzen: Ihnen genügt nicht ein einzelnes äußeres Verhalten -, sie wollen den Bürger, also den Menschen bewegen, sich zum Bürger zu steigern und als solcher in die Allgemeinheit einzugliedern. Sie befehlen also nicht, sondern sie werben, sie wollen ansprechen und gewinnen.

Hieraus folgt als erstes, daß selbst der scheinbar technischste Satz einer Verfassung niemals ausschließlich technische Bedeutung haben kann, daß vielmehr auch er Bestandteil einer Verfassung ist, die sich Nationale Integration als Ziel gesetzt hat. Auf der anderen Seite gibt es Verfassungssätze, die eines rechten technischen Sinnes entbehren, also etwa die Außerungen, die die Staatssymbole betreffen. Wenn aber jeder Verfassungssatz weniger oder mehr dem Dienst an der Nationalen Einigung gewidmet ist, dan folgt hieraus, daß er diesem seinen Sinn entsprechend gewertet werden muß, daß also selbst eine vorwiegend technische Vorschrift nicht so stilisiert sein darf wie etwa die Bestimmungen eines Erbrechts ${ }^{72}$, daß ferner die Auslegung dies zu berücksichtigen hat und daß schließlich die Verfassungsgerichtsbarkeit solchen politischen Charakter nicht in einen juristischen verwandeln darf.

Erst in solchem Lichte werden entscheidende Verfassungssätze vollständig und richtig gesehen. Das gilt etwa für das Staatsoberhaupt: Es wird wesentlich verstanden erst, wenn man neben seinen geschriebenen Kompetenzen, die ja sehr mager zu sein vermögen, seine ungeschriebene Ratio essendi stellt: Wie immer wieder hervorgehoben wird, ist er dasjenige Staatsorgan, das die Einheit der Nation zu repräsentieren und $\mathrm{zu}$ pflegen hat, das sich also am wenigsten mit irgendeiner Besonderheit, von einer Partei angefangen, identifizieren darf. Völlig vernachlässigt scheint

68 Insbesondere für die neuen Nationen in Ưbersee; vgl. oben.

69 Verfassung und Verfassungsrecht (1928), jetzt in: Staatsrechtliche Abhandlungen, 2. Aufl. (Berlin 1968) S. $119 \mathrm{ff}$.

70 Hierzu auch Frau Bandaranaike bei Wilson a. a. O. (oben Anm. 7), S. 235: Sie nennt „the oneness of the nation" als das erste von drei Zielen, die eine Verfassung zu verfolgen habe; vgl. dort auch $\mathrm{S}$. $248 \mathrm{f}$. $71 \mathrm{Vgl}$. oben.

72 Vgl. hierzu den Wahlen-Bericht, S. 35: Verfassungssätze - zu weit gehende Abstraktion $-z u$ wenig Integration. 
die Nation-integrierende Funktion von jener Deutung der Grundrechte, die sie sich in der Abwehr des Staates erschöpfen läßt. Da in einem freiheitlichen Staat auch die Nationale Integration eine freiheitliche sein muß (wenn sie nicht überhaupt allein unter solchem Vorzeichen echte und haltbare Ergebnisse verspricht), müssen alle verfassungsrechtlichen Gewährleistungen von Freiheiten als Einbeziehung ihrer Adressaten in den Integrationsprozeß verstanden werden - als Aufforderung, sich frei an diesem Prozeß zu beteiligen und ebenso frei Verantwortung für sein Gelingen $\mathrm{zu}$ übernehmen. Es ist nicht $\mathrm{zu}$ ersehen, wie anders man sonst die Forderung nach "Partizipation“ aller an allem begründen und rechtfertigen könnte. Ist dies richtig, dann ist das Verfassungsrecht für alle Bürger jederzeit aktuell, während der überwiegende Teil der Bestimmungen des Bürgerlichen usw., vor allem aber des Strafrechts für die meisten in potentia verbleibt.

\section{Die Verfassung als Programm der Nationalen Repräsentation ${ }^{73}$}

War für das Verfassungsthema „Nationale Integration“ hier vor allem herauszuarbeiten, daß es sich um ein solches, und zwar um ein sehr wesentliches Thema handelt, so gilt dies erst recht für das Verfassungsthema „Nationale Repräsentation“: Anders als im Falle von "Integration" ist man sich hier noch nicht einmal über den Begriff einig, und zwar insbesondere nicht über diejenige seiner möglichen Bedeutungen, die die für die Staats- und Verfassungstheorie entscheidende ist. So gut wie ausnahmslos nämlich hält man dieses Institut nach wie vor für nicht mehr als einen Kunstgriff ${ }^{74}$. Man übersieht diejenige Stelle einer modernen Verfassung, die er sachgerecht $\mathrm{zu}$ besetzen hat. Es handelt sich hierbei um jene Gesinnung, die mit dem, was sie hat, niemals zufrieden ist, vielmehr unablässig auf unendliche Mehrung, Verbesserung usw. usw. ihrer Lebens-, und zwar vor allem ihrer Genußmöglichkeiten ausgeht. Man kennt die Vokabeln, die diese Gesinnung kennzeichnen, vor allem „Wachstum“, „Erhöhung des Sozialprodukts“, „mehr Wohlstand für mehr Menschen“, „Revolution der steigenden Erwartungen“ usw. Wenn man nicht annehmen will, daß alle diese Forderungen sich von selbst erfüllen oder daß jedenfalls der einzelne Mensch für sich allein imstande sei, sie zu befriedigen, dann bleibt nichts anderes übrig als die Folgerung, daß hier nur eine kollektive Anstrengung diejenigen unvergleichen Ergebnisse in Aussicht stellen kann, die die Erfüllung solch unerhörter Forderungen voraussetzt. Dies alles ist nur denkbar, wenn alle Gruppen, an der Spitze aber die Allgemeinheit, sich in den Stand setzen, immer neue Höchstleistungen hervorbringen zu können. Es ist die Verfassung, die für eine solche Leistungsfähigkeit zu sorgen hat. Dieses Thema wird hier durch einen spezifischen Begriff der Repräsentation bezeichnet: Es ist „Aufbereitung “, „Verbesserung“, „Vergütung“ u. ä. m., was hiermit gemeint ist ${ }^{75}$. Die Ver-

$73 \mathrm{Vgl}$. hierzu meinen gleichnamigen Beitrag zu der Festschrift für Ernst Rudolf Huber (Göttingen 1973), S. $95 \mathrm{ff}$.

$74 \mathrm{Vgl}$. soeben wieder F. Ossenbühl, Welche normativen Anforderungen stellt der Verfassungsgrundsatz des demokratischen Rechtsstaates an die planende staatliche Tätigkeit, dargestellt am Beispiel der Entwicklungsplanung? Gutachten B zum 50. Deutschen Juristentag (München 1974), S. B 119: ${ }_{n}$ Mit ihm (sc dem Begriff der Repräsentation) wird der Sachverhalt umschrieben, daß in den Demokratien der modernen Fegriff der Repräsentation) wird der Sachverhalt umschrieben, daß in den Demokratien der modernen Herrschenden durch die egalitäre (auf Gleichheit und Mehrheitsentscheidung beruhende) Repräsentation in einen wirksamen Legitimierungs- und Kontrollzusammenhang zu den Beherrschten gebracht werden."

75 Vgl. Herbert Krüger, Allgemeine Staatslehre, 2. Aufl. (Stuttgart 1966), S. 232 ff., S. 234 ff. 
fassung ist unter solchem Vorzeichen ein Programm, gemäß dem eine Nation sich zu höchster Leistungsfähigkeit in jeder Hinsicht immer wieder zu steigern hofft.

In diesem Lichte muß die Staatlichkeit einer Nation als der Überzeugung entsprungen angesehen werden, daß die Nation nur in dieser Gestalt sich zu jener Handlungs- und Leistungsfähigkeit zu bringen vermag, zu der sie sich gewissermaßen selbst verurteilt hat, als sie sich für unendliches Wachstum usw. entschied. Es folgt hieraus, daß sich eine Verfassung unter dem Thema „Repräsentation“ als erstes mit der Staatlichkeit der Nation zu befassen hat. Diesem Erfordernis ist nicht schon dadurch genüge getan, daß die Verfassung ihren Staat auf den Typ des Rechtsstaates, des Justizstaates oder des Sozialstaates festlegt: Keiner dieser Typen hat es mit Leistung, Ergiebigkeit, Fruchtbarkeit und dergl. zu tun, wohl aber setzen sie, und dies gilt vor allem für den Sozialstaat, alles dies ihrerseits voraus. Sind aber Handlungs- und Leistungsfähigkeit in erster Linie von der Entscheidungskraft des Gemeinwesens abhängig, dann müssen Wahl, Bildung und Einsatz der Sätze, Institute und Institutionen auch unter diesem Blickwinkel erfolgen. Um was es sich hierbei handelt, und daß die Verfassunggeber in der Tat demgemäß vorgehen, zeigt die repräsentative Demokratie: Man entscheidet sich für sie, weil sie im Vergleich zur unmittelbaren Demokratie als die in jeder Hinsicht, also auch bezüglich Leistungsfähigkeit, bessere Demokratie angesehen wird; das repräsentative Mandat wird dem imperativen vorgezogen, weil der Abgeordnete als der, der Konzeption nach bessere Mann nicht unter die Herrschaft der Wähler als der weniger guten Männer gestellt werden darf und weil gerade heute Entschlüsse nicht auf Instruktionen warten können; die Bildung der Regierung überläßt man einem solchen Parlament, weil man hofft, daß die Besten des Landes nur die Allerbesten in die Regierung entsenden werden, und zwar wie es sich gehört die allerbesten Politiker. Insgesamt gesehen muß somit eine jede Verfassung darauf bedacht sein, daß die Nation nicht nur einen einheitlichen, sondern vor allem einen richtigen Willen bildet, $d$. h. denjenigen Willen, der die Auseinandersetzung mit den Lagen mit der Aussicht auf den bestmöglichen Erfolg zu führen verspricht. $\mathrm{Daß}$ dies gerade für die neuen Nationen in Übersee gilt, liegt auf der Hand.

Die wesentlich verstandene repräsentative Demokratie war nur ein Beispiel dafür, $\mathrm{da} ß$ und wie ein Verfassunggeber die Verfassung als Programm der Nationalen Repräsentation gestalten kann. Es versteht sich von selbst, daß dies nicht die einzig mögliche Lösung ist, wenn auch noch immer allzu viele Freunde der freiheitlichen Demokratie einem entsprechenden ideologischen Monopolanspruch huldigen. Man wird z. B. auch den kommunistischen Verfassungstyp unter diesem Blickwinkel zu sehen haben, etwa die Diktatur des Proletariats, den Primat der einen Partei und die Herrschaft der Führung über diese Partei.

Integration und Repräsentation der Nation setzen ein Verständnis der Verfassungsstruktur und -dynamik voraus, von dem mit einigen Worten noch die Rede sein muß. Beide Veranstaltungen haben von vornherein einen Bezug auf die Gesellschaft: Ist hier auch zunächst einmal und gerade der einzelne gefordert, so doch nicht um einzelner zu bleiben, sondern um über sich selbst hinauszukommen, indem er frei an Bildung und Steigerung der Leistungsfähigkeit des Gemeinwesens teilnimmt. Mit dieser freien Geselligkeit des Menschen und Bürgers verträgt sich nicht ein Verständnis der Verfassung als Umschreibung und Abgrenzung von $\mathrm{Zu}$ ständigkeiten und subjektiven Rechten, sei es innerhalb des Staates selbst, sei es im Verhältnis zwischen Staat und Bürger. Gerade diese Auffassung scheint jedoch 
nicht sterben zu können - wie die Häufigkeit der Vokabeln „Trennung“, „Einschränkung76", „Hemmung“, „Abwehr“ zeigt. In diesem Rahmen scheint insbesondere die Auffassung unausrottbar, die in der Gewaltenunterscheidung lediglich Teilung und Vereinzelung zu erkennen vermag. In Wahrheit meint die Gewaltenteilung Zusammenwirken in einer Verschiedenheit und Selbständigkeit, die nicht verneint, sondern bekräftigt werden - aber eben Zusammenwirken! Es ist unmöglich, eine Verfassung lediglich als Juxtaposition von Amts- oder Individualspähren zu begreifen, aus denen heraus von ihren Inhabern ohne Rücksicht aufeinander individuell operiert wird, um von Zusammenarbeit ganz zu schweigen.

\section{Die Verfassung als Thema ihrer selbst}

Eine jede Verfassung hat sich schließlich und vor allem mit sich selbst zu beschäftigen, der Verfassunggeber hat sie als Verfassung zum Gegenstand seiner Uberlegungen zu machen. Eine jede Verfassung nämlich will und muß sein eine Nationale Kraft, eine „idée force“ oder wie immer man diese ihre Eigenart und Wirksamkeit bezeichnen mag. Was hiermit gemeint ist, liegt etwa in der Richtung der Unterscheidung, die Walter Bagehot ${ }^{77}$ zwischen den „dignified parts“ und den „efficent parts" einer Verfassung getroffen hat: "There are two great objects which every constitution must attain to be successful, which every old and celebrated one must have wonderfully achieved: every constitution must first gain authority, and then use authority; it must first win the loyalty and confidence of mankind, and then employ that homage in the work of government."

a) Im gegenwärtigen Zusammenhang bedarf es als ersten der Darlegung, was etwa gemeint ist, wenn von der Verfassung als einer "Nationalen Kraft" gesprochen wird. Ohne weiteres läßt sich sagen, daß es sich hierbei nicht um die juristische Gewalt und die tatsächliche Macht des Staates handelt, den sie konstituiert —, daß vielmehr hiermit eine eigenständige und eigenartige Energie angesprochen ist. Mit der Kraft, die einer Verfassung innewohnen und die sie zur Geltung bringen muß, ist eine geistige oder moralische Kraft gemeint - , die eigene Mächtigkeit einer Verfassung kann nur ihr Geist ${ }^{78}$ und ihre Moral sein. Beides, Geist und Moral, sind nicht dasselbe wie etwa der sublimierte Inhalt der Verfassungsätze und Verfassungsprinzipien -, diese sind lediglich Anzeichen, die auf Geist und Moral hindeuten. Diese Gebilde mögen ferner Elemente dessen sein, was man neuerdings als „Political Culture“ beschreibt: Aber es handelt sich jedenfalls um einen selbständigen, aktiven Bestandteil dieser Gesamterscheinung, der wesentlich für die Bildung einer solchen Kultur ist. Dagegen dürfte nichts im Wege stehen, die "Gestimmtheit" einer Verfassung79 oder deren "Geistige Grundlagen“ in den Geist einer Verfassung einzubeziehen oder jedenfalls als Indizien für einen solchen Geist und seine Beschaffenheit zu verwerten.

Mehr als eine solche Annäherung an eine Definition läßt sich einstweilen, wenn nicht überhaupt, nicht bieten: Geist und Moral einer Verfassung machen sich

$76 \mathrm{Vgl}$. oben bei Anmerkung 60; ferner etwa das Bundesverfassungsgericht der Bundesrepublik Deutschland vom 31.7. 1973 in BVerfGE 36.14: Wesen einer rechtsstaatlichen Ordnung jeder Politik, auch im Bereich der Auswärtigen Politik, rechtliche Schranken zu setzen. - So richtig dies ist, so kann etwas Derartiges doch nicht das einzige und letzte Wort über das Wesen einer Verfassung sein.

77 The English Constitution (zuerst 1867); hier ist benutzt die Ausgabe in "The World Classics (London Oxford UP $1928 \mathrm{ff}$.), S. 4.

78 Montesquieu versteht unter dem „esprit des lois“ die Gesamtheit der „rapports ${ }^{\alpha}$, in denen die Gesetze zu ihren Vorgegebenheiten stehen; "vgl. Oeuvres Complètes (Bibliothèque de la Pléiade) II, S. 238.

$79 \mathrm{Vgl}$. oben Anmerkungen 60 und 62. 
erst dann bemerkbar, wenn sie auf die Probe gestellt wird: Dann erst zeigt es sich, "was in ihr steckt".

b) Mit dem letzten ist die Frage angeschnitten, warum eine jede Verfassung eines "Geistes" oder einer "Moral" bedarf und schon der Verfassunggeber hierauf Bedacht zu nehmen hat. Es handelt sich einmal um Geltung und Durchsetzungsvermögen der Verfassungen: $\mathrm{Da}$ eine Verfassung im ganzen und im einzelnen ihre Adressaten $^{80}$ in ganz anderen Absichten anspricht als jede andere Art von Rechtsnormen ${ }^{31}$, ist sie auf eine ganz andere Weise solcher Ansprache angewiesen: Wirken die meisten Normen auf ihre Adressaten durch das, „was hinter ihnen steht“, so kann eine Verfassung nur durch das auf ihre Adressaten einwirken, „was in ihr steckt“; ihre Sätze und Prinzipien müssen sich selbst durchsetzen. Sie muß also darauf bedacht sein, sich durch sich selbst Achtung, Anerkennung, ja Ehrfurcht $\mathrm{zu}$ verschaffen. Insbesondere als Programm der Nationalen Integration und Repräsentation vermag eine Verfassung nur durch ihre moralische Kraft erfolgreich zu wirken: Setzt sie auf eine frei aufgenommene und dargebotene Mitwirkung möglichst aller Bürger, so muß sie eine Kraft sein, die Widerhall zu wecken sowie Initiative, Phantasie und Freude an freier Mitwirkung zu entfachen vermag.

Der Verfassung als nationaler Kraft bedarf es aus einem zweiten Grunde, der früher bereits angeklungen ist. Soll eine Verfassung einem Volke die Möglichkeit sichern, äußere und innere Tiefpunkte seiner Existenz ohne äußere Verluste und ohne inneren Schaden durchzustehen und zu überwinden, dann kann sie derartiges nur leisten, wenn sie eine Kraft ist, die nicht nur die Verfassung in solchen Tiefpunkten wahrt, sondern auch die Bürger zu stärken vermag. Eine VerfassungsUrkunde ist gewiß nicht eine Art von Heiliger Schrift, deren Wort aufrichtet. Aber nicht ganz unähnlich einer solchen soll sie den Bürgern inneren Halt vermitteln, wenn sie etwa eines solchen bedürfen - wozu angemerkt werden darf, daß ein Bedürfnis hiernach nicht nur in Krisen, sondern immer besteht.

c) Als dritte und schwierigste stellt sich die Frage, wie eine Verfassung eine solche Kraft sein, d. h., wie sie sich selbst zu einer solchen Fähigkeit verhelfen kann. Die Verfassunggebung vermag, wie früher gezeigt worden ist ${ }^{82}$, entscheidendes beizutragen, wenn sie zur rechten Zeit und in hoher Gestimmtheit erfolgt. Sie mag ferner durch Instituierung entsprechender Veranstaltungen wie z. B. politischer Feste dafür sorgen, daß der Geist lebendig und gegenwärtig bleibt.

Dies vorausgesetzt ergibt sich als erste, selbstverständliche Erkenntnis, daß eine Verfassung nicht nur ihre eigene Denaturierung und Degradierung nicht ins Auge fassen darf - daß sie vielmehr auf jeden Fall Vorkehrungen dagegen zu treffen hat, daß die Praxis sie denaturiert oder degradiert. Als Beispiel für das erste sei eine Ermächtigung genannt, wie sie etwa den Kriegsdienstverweigerern heute überall zuteil wird - die Ermächtigung nämlich, Verfassung und Gesetz nach eigenem Gutdünken für sich selbst außer Verbindlichkeit setzen zu dürfen. Ist dies schon schädlich genug, so rührt es an die Wurzeln der Verfassung als geistiger und moralischer Kraft, wenn solche Selbstdispensierung und Selbstprivilegierung gerechtfertigt werden darf aus dem Gewissen. Hiermit bekundet ein solcher Bürger seinen, die allgemeine Wehrpflicht erfüllenden Mitbürgern, daß sie ein weniger empfindliches Gewissen haben als er; die Erfüllung einer allgemeinen Bürgerpflicht 80 Vgl. hierzu Herbert Krüger, Der Adressat des Verfassungssatzes, in: Festschrift für Dendrias.
81 Vgl. hierzu auch oben.

$82 \mathrm{Vgl}$. oben. 
wird sittlich geringer gewertet als die Nicht-Erfüllung. Entsprechend läßt sich die Verfassung kraft ihres eigenen Willens von Bürgern bescheinigen, daß sie und die verfassungsmäßigen Gesetze insoweit kein rechtes Gewissen haben. Der klassische Verfassungsstaat hat eine solche Konstellation uind die dadurch bewirkte Schwächung von Geist und Moral der Verfassung dadurch vermieden, daß er sich als ausschließlich weltlicher Staat verstand und es im Vertrauen hierauf seinen Bürgern zu verwehren pflegte, sich unter Berufung auf widerstreitende "höhere" Úberzeugungen selbst von ihren staatlichen Verpflichtungen $\mathrm{zu}$ entbinden ${ }^{83}$. Ferner schließt dieser Staat nicht zuletzt um seiner geistigen und moralischen Kraft willen Privilegierungen aus, weil sie bei den Nicht-Privilegierten Staatsverdrossenheit erzeugen müssen, und zwar vor allem wegen des von ihnen geschaffenen Parasitentums: Niemand zahlt gern Steuern, damit "glücklichere“ Mitbürger keine Steuern $\mathrm{zu}$ entrichten brauchen ${ }^{34}$. Sollte einmal Dispensierung oder Privilegierung ganz unvermeidlich sein, dann sollte sie um der Verfassung als nationaler Kraft willen nicht durch Verfassungssatz, sondern durch einfaches Gesetz erfolgen.

Sich selbst degradiert eine Verfassung, die sich nicht auf die Aufnahme von "Materiellem Verfassungsrecht" beschränkt ${ }^{85}$. Was nämlich in den Rang von formellem Verfassungsrecht erhoben wird, ist dieser Rangerhöhung nicht würdig, senkt also die Verfassung insoweit auf die Ebene einfacher Gesetze ${ }^{86}$. Umgekehrt sollte alles materielle Verfassungsrecht Aufnahme in die Verfassungsurkunde finden, damit nicht der Widerspruch zwischen hohem Gehalt und niederer Form zur Degradierung der Verfassung beiträgt.

Es scheint nicht allzuviel zu sein, was die Verfassung selbst gegen Denaturierung und Degradierung zu tun vermag. Um so stärker wird hierdurch die Verantwortung der Verfassungspraxis. Indem man die staatlichen Normen in eine Hierarchie ordnet und die Verfassung an deren Spitze setzt, schafft man die Gefahr der Banalisierung, indem nunmehr der Durchgriff von unten nach oben jedenfalls zulässig, wenn nicht sogar Pflicht ist. Es ist die Verfassungsbeschwerde, die diese Gefahr vor allem zu einer Realität macht. Zwar ist es schön, wenn der Bürger auch im Alltag mit der Verfassung lebt und webt. Aber diese Allgegenwärtigkeit soll gewiß nicht die Banalität zum Zuge bringen, also etwa die künstliche Besamung von Rindvieh durch einen Tierarzt zu dessen Persönlichkeitsentfaltung im Sinne des Art. 2 des Grundgesetzes hochstilisieren. Alles dies gilt insbesondere dann, wenn die Banalität die Grenzen der Lächerlichkeit streift, wenn nicht sogar überschreitet. Ferner sind die Grundrechte gewiß dazu bestimmt, den Bürger gegen Fehler von Verwaltung und Gesetzgebung zu schätzen. Aber dieser die Fehlsamkeit des Staates abwehrende Gebrauch darf nicht zur Abwehr des Staates überhaupt entarten: Eine gewisse Proportion muß also hier immer gewahrt bleiben - die Abwehr des richtig handelnden Staates darf nicht die Hauptsache des Grundrechtsgebrauches ausmachen, zumal wenn man an Integration und Repräsentation der Nation denkt. Positiv vermag eine Verfassung für ihre nationale Mächtigkeit vor allem dadurch zu sorgen, daß sie sich auf hohem und höchstem Niveau in jeder Beziehung be-

83 Typisch z. B. die Bundesverfassung der Schweizerischen Eidgenossenschaft von 1874 Art. 49 Abs. V: „Die Glaubensansichten entbinden nicht von der Erfüllung der bürgerlichen Pflichten."

84 Dies gilt natürlich erst recht für den Wehrdienst, und deshalb hat der Staat des 19 . Jahrhunderts alle Exemtionen beseitigt, mochten sie rechtlich auch noch so wohl gegründet sein; vgl. hierzu F. K. Fürst Hohenlohe-Waldenburg, Das Verfahren der Königlich Württembergischen Regierung gegen die Standesherren (Karlsruhe 1867).

85 Vgl. oben bei Anmerkung 63

86 Ein klassisches Beispiel hierfür bietet das Absinth-Verbot des Art. 32 ter der Schweizerischen Bundes- 
wegt, also insbesondere nicht eine Verfassung von Kleinbürgern für Kleinbürger, sondern von Weltmännern für Weltmänner ist. Hierher gehört als erstes die Normativitität einer Verfassung: Macht sie mangels solcher Normativität alle Niveauschwankungen der politischen Wirklichkeit mit, dann verfehlt sie sich nicht nur gegen diese Wirklichkeit, sondern vor allem auch gegen sich selbst. Zweitens: Wir haben das von einer Verfassung zu haltende und $\mathrm{zu}$ wahrende Niveau durch das Stichwort „Welt" veranschaulicht. Hierher gehört vor allem, daß alle Verfassungsbeteiligten jedenfalls entschlossen sind, sich als Weltmänner $\mathrm{zu}$ verstehen und $\mathrm{zu}$ verhalten, und zwar vor allem auch der schlichte Grundrechtsträger: Die Mitwirkung an Integration und Repräsentation der Nation gibt ihm Gelegenheit, durch die Teilnahme an großen Angelegenheiten sich zum Weltmann zu erheben und sich als solcher zu bewähren, womit sich zeigt, daß „Abwehr" des Staates durch Grundrechte letztlich auf Abwehr der Welt also Provinzialismus hinausläuft. „Welt" meint drittens eine "Atmosphäre", die eine Verfassung ebenso voraussetzt, wie sie sie bestimmt: Soll die Verfassung eine "Nationale Kraft" sein, so ist damit verworfen eine Stickluft, wie sie etwa das Heilige Römische Reich und die ihm entsprechende staatsrechtliche Literatur gelähmt hat, und bejaht eine „Weltluft", für die der Verfassunggeber insbesondere durch Instituierung einer Opposition zu sorgen hat, die ständig für frischen und scharfen Wind in der Politik verantwortlich ist. Schließlich bezeichnet "Welt" den Stil des politischen Lebens ${ }^{37}$ : Was damit vor allem ausgeschlossen ist, ist das, was die deutsche Sprache volkstümlich als „kleinkariert" bezeichnet.

Lediglich hingewiesen sei darauf, daß die Wohlstandsgesellschaft alledem, was hier zum Thema "Verfassung als nationale Kraft" angedeutet worden ist, alles andere als förderlich ist: Ihre "Ideale“ sind durchweg nicht von demjenigen Niveau, das eine solche Kraft bedingt. Es genügt, auf die Lektüre der Wohlstandsgesellschaft hinzuweisen. In dieser Ambiance muß sich eine Verfassung als nationale Kraft bewähren, ohne daß ihr solche Kraft zugeführt würde: Eine beinahe unlösbar scheinende Aufgabe.

\section{Die Menschen- und Bürgerrechte als Gegenstand der Verfassunggebung}

Vielleicht wird man kritisieren, daß die Menschen- und Bürgerrechte bisher nicht als notwendiges Thema einer jeden Verfassung als Verfassung vorgeführt worden sind. Es erklärt sich dieser Umstand daraus, daß sie nicht zu solchen Gegenständen gehören oder jedenfalls in deren Abfolge nicht an der Spitze stehen. Vor allem wenn man die Grundrechte als Abwehrrechte (miß)versteht, muß offenbar das Gemeinwesen erst einmal konstituiert sein, ehe man seine Außerungen abwehren kann. Begreift man sie richtig, dann bedarf es zuerst der Festlegung und der Verfassung eines Staatstypes, der Integration und Repräsentation des Gemeinwesens der freien Mitwirkung seiner Bürger anvertraut ${ }^{88}$. Die Notwendigkeit dieses Themas erweist sich für jede der beiden alternativen Grundrechtsauffassungen als eine relative. Eben deswegen soll dieser Gegenstand der Verfassunggebung als ein möglicher nicht weiter verfolgt werden.

verfassung; hierzu J.-F. Aubert, Petite Histoire Constitutionelle de la Suisse (Bern 1974).

$87 \mathrm{Daß}$ dieser in der Tat erheblich ist, zeigt die Gewohnheit deutscher Politiker, sich gegenseitig "schlech-

$88 \mathrm{Für}$ die besonderen Gegebenheiten (Denken in Gruppen), in denen sich insoweit die neuen Staaten Afrikas vorfinden, vgl. P. F. Gonidec, Les Systèmes politiques Africaines: Deuxième Partie - Les Réalités du Pouvoir - (Paris 1974), S. $50 \mathrm{ff}$. 


\title{
The Art of Constitution-Making
}

\section{By Herbert KrüGer}

In 1973 a commission preparing a total revision of the Swiss Federal Constitution published a report („Wahlen-Bericht"). The first part of this report, which deals with the conditions for a total revision of the Federal Constitution, could be called an instruction for constitution-making. The pattern of constitution-making has first of all to deal with the mode of procedure. But for lack of material the author is not able to draw any generalisation in this field. Therefore he attempts to show how the rightness of the contents of a constitution may be achieved. This depends on the situation in which constitution-making is attempted. Two main situations are distinguished, the ending of a revolution and constitutional change in an undisturbed state. After a revolution, the constitution has to establish a political order and to build a nation, whereas in the latter case continuity must be regarded as a high priority.

The constitution is shaped by the ideas of the constitution-maker and his perception of the realities of his country. It seems that a good constitution has to rely more on the social reality than on ideologies. Therefore it is necessary, that the constitution-maker analyzes the concrete situation of his state. The constitution however should not be a transformation of the reality into norms only, but should also achieve a better society. It is clear, that a constitution-maker will generally fail, if he is guided by mistrust and pessimism. The two main features of a constitution are: a programme of national integration and a programme of national

representation. Integration means the building of a nation by integration of the individuals as citizens into the state. A constitution has to provide the possibilities for this process, namely the symbols of integration and the freedoms, which are necessary to perform the role of a citizen. Representation means that the citizens must form a collective will, for only collective efforts can satisfy the great needs in our times. The constitution has to provide also for the formulation of a common will and a procedure, which guarantees that not any will but the best or at least the better will may prevail. Finally the constitution has to be a national authority in order to prohibit its destruction. This is the most difficult task of constitutionmaking. A constitution can gain the necessary authority if it relies not on private ideals but on cosmopolitan ideals of the highest standard.

\section{The Constitution of Bangladesh and a Short Constitutional History}

\author{
By S. C. SEN
}

After the withdrawal of the British from the Indian Empire Bengal found itself as part of Pakistan, but the only link with West-Pakistan (1200 miles away) was religion. In 23 years of joint history the East was denied almost all rights. With the six-point formula of Bengal autonomy Sheikh Mujibur Rehman gained an enormous victory in the 1970 election to the proposed National Assembly. Fearing majorisation by the East the party leaders of the West secured the postponement of the meeting of the National Assembly by President Yaya Khan. Over a campaign of non-cooperation answered by a reign of terror, this led to the Declaration of Independence and the civil war. 